\title{
Measurement of Neutrino Oscillations with Neutrino Telescopes
}

\author{
Jürgen Brunner \\ CPPM, Aix-Marseille Université, CNRS/IN2P3, Marseille, France \\ Correspondence should be addressed to Jürgen Brunner; brunner@cppm.in2p3.fr
}

Received 11 August 2013; Accepted 27 September 2013

Academic Editor: Elisa Bernardini

Copyright (C) 2013 Jürgen Brunner. This is an open access article distributed under the Creative Commons Attribution License, which permits unrestricted use, distribution, and reproduction in any medium, provided the original work is properly cited.

\begin{abstract}
IceCube and ANTARES are the world-largest neutrino telescopes. They are successfully taking data, producing a wealth of scientific results. Whereas their main goal is the detection of cosmic neutrinos with energies in the TeV-PeV range, both have demonstrated their capability to measure neutrino oscillations by studying atmospheric neutrinos with energies of $10-50 \mathrm{GeV}$. After recalling the methods of these measurements and the first published results of these searches, the potential of existing, and planned low-energy extensions of IceCube and KM3Net are discussed. These new detectors will be able to improve the knowledge of the atmospheric neutrino oscillation parameters, and in particular they might help to understand the neutrino mass hierarchy. Such studies, which use atmospheric neutrinos, could be complemented by measurements in a long-baseline neutrino beam, which is discussed as a long-term future option.
\end{abstract}

\section{Introduction}

The main goal of neutrino telescopes such as IceCube [1] and ANTARES [2] is the observation of high-energy neutrinos from nonterrestrial sources. These telescopes are optimised for the detection of Cherenkov light induced by charged particles issued from neutrino interactions at $\mathrm{TeV}$ energies. To cope with the expected feeble neutrino fluxes at these energies, target masses of up to one Gton are equipped with low-density arrays of photomultipliers. Such a low-density of detector elements implies a rather high energy threshold for neutrino detection of about $50 \mathrm{GeV}$.

Nevertheless both IceCube (exploiting its low-energy infill DeepCore [3]) and ANTARES developed dedicated analysis methods to access neutrino energies as low as $20 \mathrm{GeV}$, where the effect of neutrino oscillations starts to be measurable and both collaborations published recently first results on the measurement of parameters for atmospheric neutrino oscillations.

Two principal event signatures can be distinguished. Track-like events are characterized by the presence of a long muon track, which can be identified by the light pattern from Cherenkov light emitted along its path. Such events are the result of muon neutrino charged current (CC) interactions or tau neutrino CC interactions followed by a muonic tau decay in conjunction with an analysis-dependent condition on the minimal muon energy. Cascade-like events are distinguished by the absence of such a long muon track. The low density of detector elements does not allow to separate hadronic from electromagnetic cascades. Therefore all remaining reaction channels such as neutral current (NC) reactions, electron and tau neutrino CC interactions (the latter followed by nonmuonic tau decays) are commonly called cascade-like events or just "cascades".

First, published results of ANTARES and IceCube are presented, followed by the discussion of very recent and still preliminary studies and their long-term potential. The second part of the paper is dedicated to the planned low-energy extensions of neutrino telescopes in the Mediterranean Sea as well as in the Antarctic ice. Their potential of studying atmospheric neutrinos is discussed. The final section evokes the possibilities of sending neutrino beams towards these future neutrino telescopes.

\section{Atmospheric Neutrino Oscillations at $E_{\gamma}>15 \mathbf{G e V}$}

At energies of $E_{v}>15 \mathrm{GeV}$, Earth matter effects can be ignored because the resonance energies for typical Earth densities are significantly lower (see Section 7 and Figure 6). Oscillation effects are prominently seen in the suppression of 
muon neutrinos. The survival probability of atmospheric $\nu_{\mu}$ in the framework of three-flavour mixing is given as

$$
\begin{aligned}
P\left(\nu_{\mu} \longrightarrow \nu_{\mu}\right) & 1-4\left|U_{\mu 1}\right|^{2}\left|U_{\mu 2}\right|^{2} \sin ^{2}\left(\frac{1.27 \Delta m_{21}^{2} L}{E_{\nu}}\right) \\
& -4\left|U_{\mu 1}\right|^{2}\left|U_{\mu 3}\right|^{2} \sin ^{2}\left(\frac{1.27 \Delta m_{31}^{2} L}{E_{\nu}}\right) \\
& -4\left|U_{\mu 2}\right|^{2}\left|U_{\mu 3}\right|^{2} \sin ^{2}\left(\frac{1.27 \Delta m_{32}^{2} L}{E_{\nu}}\right),
\end{aligned}
$$

where $L$ is the travel path (in $\mathrm{km}$ ) of the neutrino through the earth and $E_{v}$, its energy (in $\mathrm{GeV}$ ). $U_{\alpha i}$ is the $3 \times 3$ PMNS matrix which describes the mixing between flavour eigenstates $v_{e}, v_{\mu}, v_{\tau}$ and mass eigenstates $v_{1}, v_{2}$, and $v_{3}$ and $\Delta m_{i j}^{2}=m_{i}^{2}-m_{j}^{2}\left(\right.$ in $\left.\mathrm{eV}^{2}\right)$ is the difference of the squares of the masses of the corresponding neutrino mass eigenstates. The numbering of the mass eigenstates $v_{1}, v_{2}$, and $v_{3}$ is chosen such that

$$
\left|U_{e 1}\right|^{2}>\left|U_{e 2}\right|^{2}>\left|U_{e 3}\right|^{2}
$$

For atmospheric neutrinos, $L \leq D$, where $D$ is the Earth diameter, is always satisfied. If the condition $E_{v}>15 \mathrm{GeV}$ is taken into account, the term $\sin ^{2}\left(1.27 \Delta m_{21}^{2} L / E_{\nu}\right)$ does not exceed $5 \cdot 10^{-3}$ when using $\Delta m_{21}^{2}$ from [4]. This term can safely be ignored as well as differences between $\Delta m_{31}^{2}$ and $\Delta m_{32}^{2}$ and (1) simplifies to

$$
\begin{aligned}
P\left(\nu_{\mu} \longrightarrow \nu_{\mu}\right)= & 1-4\left(1-\left|U_{\mu 3}\right|^{2}\right) \\
& \times\left|U_{\mu 3}\right|^{2} \sin ^{2}\left(\frac{1.27 \Delta m_{32}^{2} L}{E_{\nu}}\right) .
\end{aligned}
$$

Results could in principle be extracted in terms of $\left|U_{\mu 3}\right|^{2}$ and $\left|\Delta m_{32}^{2}\right|$ which are the two oscillation parameters in (3). To maintain compatibility with earlier results, a mixing angle $\sin ^{2} \theta_{23}=\left|U_{\mu 3}\right|^{2}$ is defined, ignoring the $2.4 \%$ deviation from 1 of $\cos ^{2} \theta_{13}=0.976[5,6]$. This leads to the usual two-flavour description

$$
P\left(v_{\mu} \longrightarrow v_{\mu}\right)=1-\sin ^{2} 2 \theta_{23} \sin ^{2}\left(\frac{16200 \Delta m_{32}^{2} \cos \Theta}{E_{\nu}}\right),
$$

where $L$ has been replaced by $D \cdot \cos \Theta$ with $\Theta$ the zenith angle. The transition probability $P$ depends now on only two oscillation parameters, $\left|\Delta m_{32}^{2}\right|$ and $\sin ^{2} 2 \theta_{23}$, which determine the behaviour for the atmospheric neutrino oscillations. With $\left|\Delta m_{32}^{2}\right|=2.32 \cdot 10^{-3} \mathrm{eV}^{2}$ and $\sin ^{2} 2 \theta_{23}=1$ from [4] one expects the first oscillation maximum; that is, $P\left(\nu_{\mu} \rightarrow\right.$ $\left.v_{\mu}\right)=0$ for vertical upgoing neutrinos $(\cos \Theta=1)$ at $E_{v}=24 \mathrm{GeV}$. Muons induced by $24 \mathrm{GeV}$ neutrinos can travel up to $120 \mathrm{~m}$ in sea water or Antarctic ice. Both ANTARES and IceCube have performed studies of neutrino oscillations using the suppression of muon neutrino events in the vicinity of this first oscillation maximum. Results of these studies are presented in the following.

\section{The ANTARES Detector}

A detailed description of the ANTARES detector can be found in [2]. The detector consists of 12 lines and equipped with photosensors and a junction box which distributes the power and clock synchronization signals to the lines and collects the data. The junction box is connected to the shore by a $42 \mathrm{~km}$ electro-optical cable. The length of the detection lines is $450 \mathrm{~m}$, of which the lowest $100 \mathrm{~m}$ are not instrumented. Their horizontal separation is about $65 \mathrm{~m}$ and they are arranged to form regular octagon on the sea floor. They are connected to the junction box with the help of a submarine using wet-mateable connectors. Each line comprises 25 storeys each separated by a vertical distance of $14.5 \mathrm{~m}$. The lines are kept taut by a buoy at the top of the line and an anchor on the seabed. The movement of the line elements due to the sea currents is continuously measured by an acoustic calibration system with an accuracy of $10 \mathrm{~cm}$ [7]. Each storey contains three $45^{\circ}$ downwardlooking $10^{\prime \prime}$ photomultiplier tubes (PMT) inside pressure resistant glass spheres-the optical modules [8]. Some of the storeys contain supplementary calibration equipment such as acoustic hydrophones or optical beacons [9].

The signals of each photomultiplier are read out by two ASICs. The charges and arrival times of the PMT signals are digitised and stored for transfer to the shore station [10]. The time stamps are synchronised by a clock signal which is sent at regular intervals from the shore to all electronic cards. The overall time calibration is better than $0.5 \mathrm{~ns}$ [11]. Therefore the time resolution of the signal pulses is limited by the transit time spread of the photomultipliers ( $\sigma \sim 1.3 \mathrm{~ns}$ ) [12] and by chromatic dispersion for distant light sources. All data are sent to the shore station. With the observed optical background rate of $70 \mathrm{kHz}$ per PMT at the single photon level, this produces a data flow of several Gbit/s to the shore. In the shore station a PC farm performs data filtering to reduce the data rate by at least a factor of 100 [13]. Several trigger algorithms are applied depending on the requested physics channel and on the observed optical noise.

\section{ANTARES Neutrino Oscillation Measurement}

This analysis is based on data taken with the ANTARES detector between March 2007 and December 2010. Until December 2007, ANTARES operated in a 5-line configuration, followed by several months of operation with 10 installed detector lines. The detector construction was completed in May 2008. All physics runs taken under normal conditions have been used. The events selected by two tight trigger conditions are used [2]. The analysed sample consists of 293 million triggers, dominated by atmospheric muons, corresponding to a detector live time of 863 days. 
Downgoing atmospheric muons were simulated with the program MUPAGE [14, 15] which provides parameterised muon bundles at the detector. Alternatively, also CORSIKA [16] was used for cross checks and systematic studies. Upgoing neutrinos were simulated according to the parameterisations from [17-19] in the energy range from $10 \mathrm{GeV}$ to $10 \mathrm{PeV}$. The Cherenkov light, produced inside or in the vicinity of the detector instrumented volume, was propagated taking into account light absorption and scattering in sea water [20]. The angular acceptance, quantum efficiency, and other characteristics of the PMTs were taken from [8] and the overall geometry corresponded to the layout of the ANTARES detector [2]. The optical noise was simulated from counting rates observed in the data. At the same time, the definition of active and inactive channels has been applied from data runs as well. The generated statistics correspond to an equivalent observation time of 100 years for atmospheric neutrinos and ten months for atmospheric muons.

The algorithm used to determine the zenith angle $\Theta_{R}$ of the muon track is described in [21]. A first step of this procedure is a strict hit selection, which aims at the selection of those Cherenkov photon hits from the muon track, which did not undergo any substantial scattering on their path. If the selected hits occur only on one detector line, a singleline fit is performed. No azimuth angle is determined in this case due to the rotational symmetry of the problem. This does not affect the present measurement, as the oscillation probability does not depend on the azimuth angle (see (4)). If selected hits occur instead on several detector lines, a multiline fit is performed which provides both the zenith and azimuth angles of the track. The inclusion of singleline events is a special feature of the reconstruction method and allows to significantly lower the energy threshold of the final atmospheric neutrino sample. Whereas for multiline events, the threshold energy of the final neutrino sample is about $50 \mathrm{GeV}$ due to the $65 \mathrm{~m}$ horizontal spacing between lines, single-line events are reconstructed down to $20 \mathrm{GeV}$ for nearly vertical tracks, accessing events with $E_{\gamma} / L$ values close to the first oscillation maximum.

The neutrino energy is estimated from the observed muon range in the detector $\left(E_{R}\right)$. The presence of a hadronic shower at the neutrino vertex is ignored, as well as the fact that the muon might leave or enter the detector, making only a fraction of its actual range available for measurement.

Downgoing atmospheric muons might contaminate the event sample of upgoing atmospheric neutrinos if misreconstructed. A cut on the quality of the reconstructed tracks is needed to reduce this contamination and derive reliably neutrino oscillation parameters from the data set. The cut is chosen in such a way that the contamination of misreconstructed atmospheric muons remains below $5 \%$. The selected event sample consists of 2126 events.

The oscillation parameters are extracted from a $\chi^{2}$ minimisation of an event distribution in $E_{R} / \cos \Theta_{R}$. Systematic uncertainties lead to correlated distortion of this distribution. They are implemented as pull factors $\epsilon$ and $\eta$ which act as independent free normalisations for the single-line, and multiline sample, respectively.
Figure 1 shows the event distribution in $E_{R} / \cos \Theta_{R}$ and the fraction of measured and simulated events with respect to the nonoscillation Monte Carlo hypothesis. A clear event deficit for $E_{R} / \cos \Theta_{R}<60 \mathrm{GeV}$ can be seen as expected assuming atmospheric neutrino oscillations.

Restricting the $\chi^{2}$ minimisation to parameters in the physically allowed region yields the red curve of Figure 1 with $\left|\Delta m_{32}^{2}\right|=3.1 \cdot 10^{-3} \mathrm{eV}^{2}$ and $\sin ^{2} 2 \theta_{23}=1.00$. The corresponding pull factors are $\epsilon=-0.138$ and $\eta=-0.142$. The fit yields $\chi^{2} / \mathrm{NDF}=17.1 / 21$. For the nonoscillation hypothesis, that is, $\sin ^{2} 2 \theta_{23}=0, \chi^{2} / \mathrm{NDF}=31.1 / 23$ is obtained. The pull factors in this case are $\epsilon=-0.302$ and $\eta=-0.196$. The event deficit in the single-line channel is seen here as $\epsilon$ becoming lower than $\eta$. Requiring in addition $\epsilon=\eta$ the $\chi^{2}$ increases further to $\chi^{2} / \mathrm{NDF}=40.0 / 24$, which has a probability of only $2.1 \%$. This measurement is converted into a $90 \%$ C.L. contour of the oscillation parameters and is shown in Figure 5. More details on this analysis can be found in [22].

\section{The IceCube Detector}

IceCube is a cubic-kilometer neutrino detector installed in the ice at the geographic South Pole [1]. It consists of 5,160 digital optical modules (DOMs). Each of them contains a photomultiplier tube, supporting hardware and electronics with in situ pulse digitization $[23,24]$ inside a pressure glass sphere. These optical modules are arranged on 86 strings frozen into the antarctic ice at depths from $1450 \mathrm{~m}$ to $2450 \mathrm{~m}$ each holding $60 \mathrm{DOMs}$. The primary (high-energy) detector has a spacing of $17 \mathrm{~m}$ between sensors and an average horizontal distance of $125 \mathrm{~m}$ between neighboring strings. The low-energy infill array DeepCore consists of eight dedicated strings with a typical spacing of $70 \mathrm{~m}$ deployed near the center of the IceCube array. On the dedicated Deep- Core strings, the sensors are concentrated in the clearest deep ice, with a denser $7 \mathrm{~m}$ vertical spacing.

Optical properties of South Pole ice are depth dependent. Typical optical absorption lengths are 100-140 $\mathrm{m}$ and typical optical effective scattering lengths are $25-35 \mathrm{~m}$. The latter limits the time resolution of the signal pulses from Cherenkov light. Unlike sea water, Antarctic ice has a low concentration in radioactive isotopes. In particular ${ }^{40} \mathrm{~K}$, abundant in salty sea water, is absent in ice. Furthermore, no light-producing biological activity occurs. Therefore, the observed optical background rate at the single photon level is only $500 \mathrm{~Hz}$ per PMT in IceCube.

Beginning with the installation of the first string in 2005, IceCube has been operated in approximately year-long data taking seasons. During the Antarctic summer seasons in December and January new strings had been deployed at a pace up to 20 strings per year. IceCube construction was finished in December 2010. From May 2010 to April 201179 strings had been active (IC79), after this period data taking proceeds with the complete detector (IC86). The results which will be presented in the following have been obtained from analysing these two data-taking periods. 


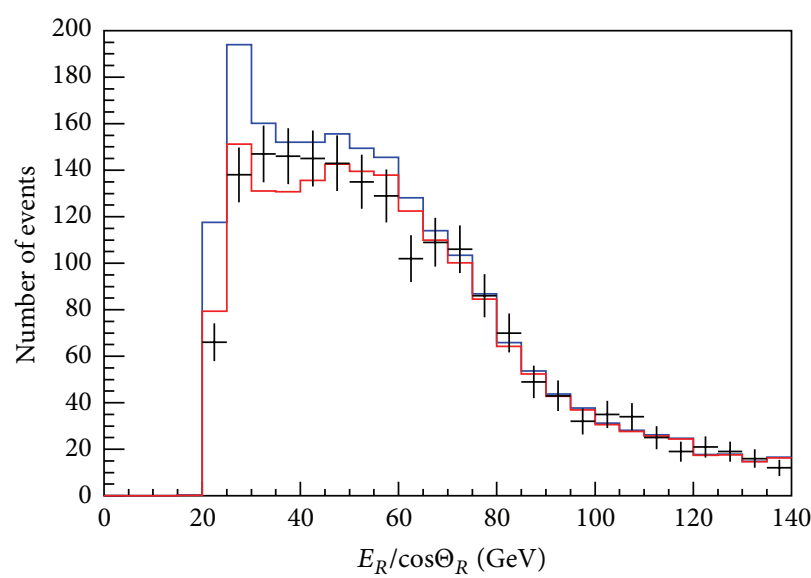

(a)

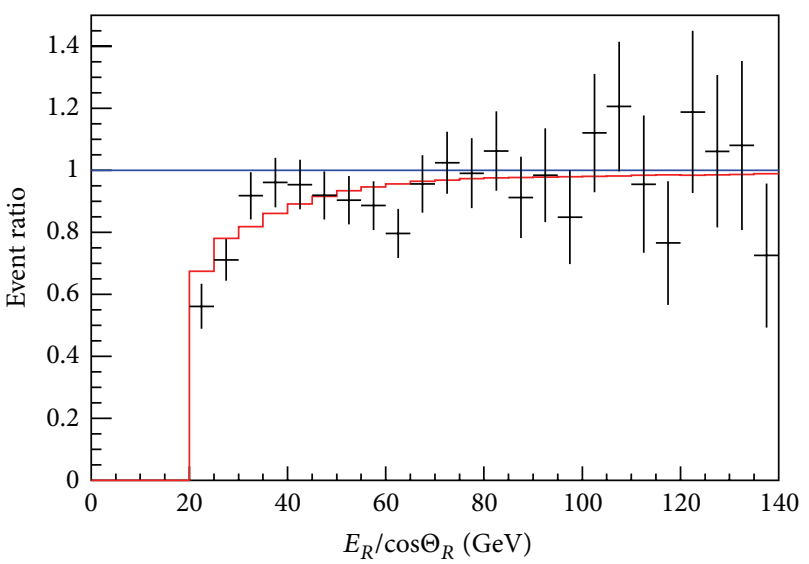

(b)

FIGURE 1: (a) Distribution of $E_{R} / \cos \Theta_{R}$ for selected events. Black crosses are data with statistical uncertainties, whereas the blue histogram shows simulations of atmospheric neutrinos without neutrino oscillations plus the residual background from atmospheric muons. The red histogram shows the result of the fit. (b) The fraction of events with respect to the nonoscillation hypothesis as function of $E_{R} / \cos \Theta_{R}$. Same color code as for the left figure (figure from [22]).

\section{IceCube Neutrino Oscillation Measurements}

Downgoing atmospheric muons were simulated with CORSIKA [16]. The flux of atmospheric neutrinos has been taken from [19]. Both the flux normalisation and the spectral index are allowed to vary and they are included as nuisance parameters in the analyses described below.

6.1. IC79 Zenith Angle Analysis. A first analysis [25] exploited two samples of upgoing muon neutrino events which had been collected during a 318.9-day period with the IC79 configuration, excluding periods of calibration runs, partial detector configurations, and detector downtime. The first sample was obtained from high-energy events using data from the entire IceCube detector. The second sample, selected from events starting in the DeepCore volume, was using the surrounding IceCube array as an active veto to reject atmospheric muon background and high-energy (>100 GeV) neutrinos [26].

The directions of the neutrino-induced muon tracks in the high-energy sample were determined with the standard maximum likelihood muon track reconstruction of IceCube [27]. For low-energy events, the same method was applied as an initial step. However, the standard hypothesis of a throughgoing track is not appropriate at low energies. In a subsequent step, the finite length of the low-energy muons which might start or stop inside the equipped detector volume was taken into account. Quality cuts on variables related to the track fit eliminated misreconstructed downgoing atmospheric muons. The final event sample consisted of 719 low-energy events, while the high-energy sample contained 39,638 events after final cuts. The simulated neutrino energy distribution of the two samples is shown in Figure 2. The high-energy sample is not affected by standard oscillations and it serves as a reference sample which absorbs systematic

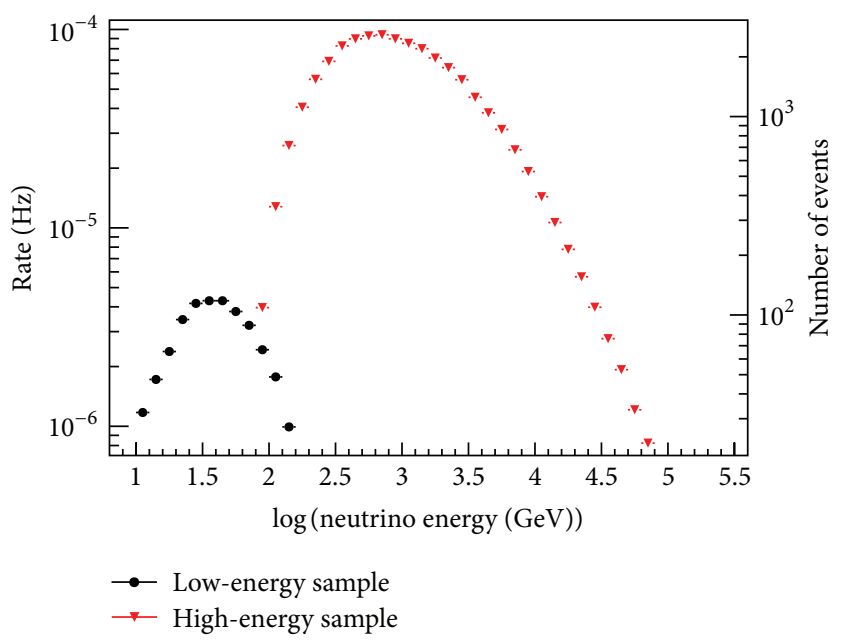

FIGURE 2: Expected distribution of the neutrino energy of atmospheric neutrinos in the low-energy (DeepCore) and in the highenergy (IceCube) samples according to simulations (figure from [25]).

effects related to the overall normalisation. Instead neutrino oscillations are expected to deplete the low-energy sample.

The oscillation parameters are extracted from the zenith angle distribution, shown in Figure 3 of the events in both samples. Within the two samples, no further use of energy is made. A $\chi^{2}$-minimization including various systematic effects is applied to the 20 bins in $\cos \Theta$ in both samples.

The fit yields $\left|\Delta m_{32}^{2}\right|=2.3_{-0.6}^{+0.5} \cdot 10^{-3} \mathrm{eV}^{2}$ and $\sin ^{2} 2 \theta_{23}>$ 0.93 at $68 \%$ C.L. The nonoscillation hypothesis can be rejected at $5.6 \sigma$. All pull factors related to systematic effects are found within one $\sigma$ from their nominal values. The resulting 90\% C.L. contour is shown in Figure 5.

Both analyses, which have been discussed so far, are final and their results have been published. Two more studies of 


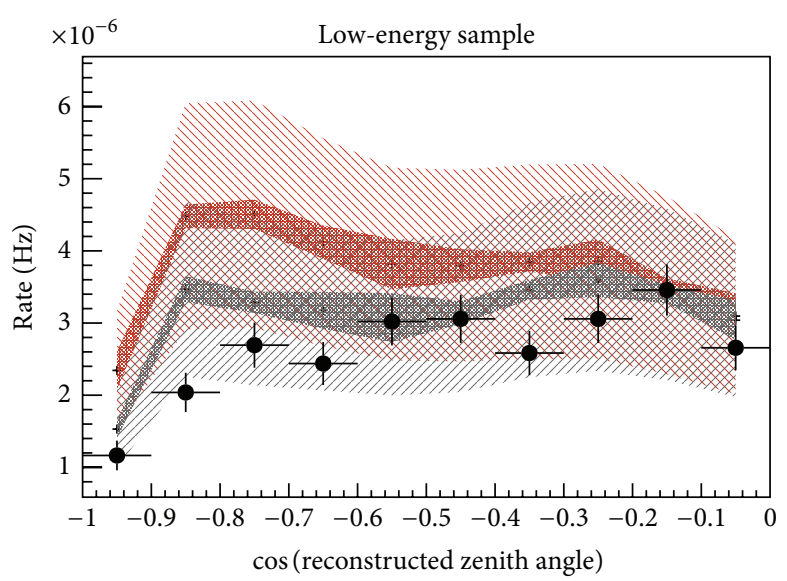

(a)

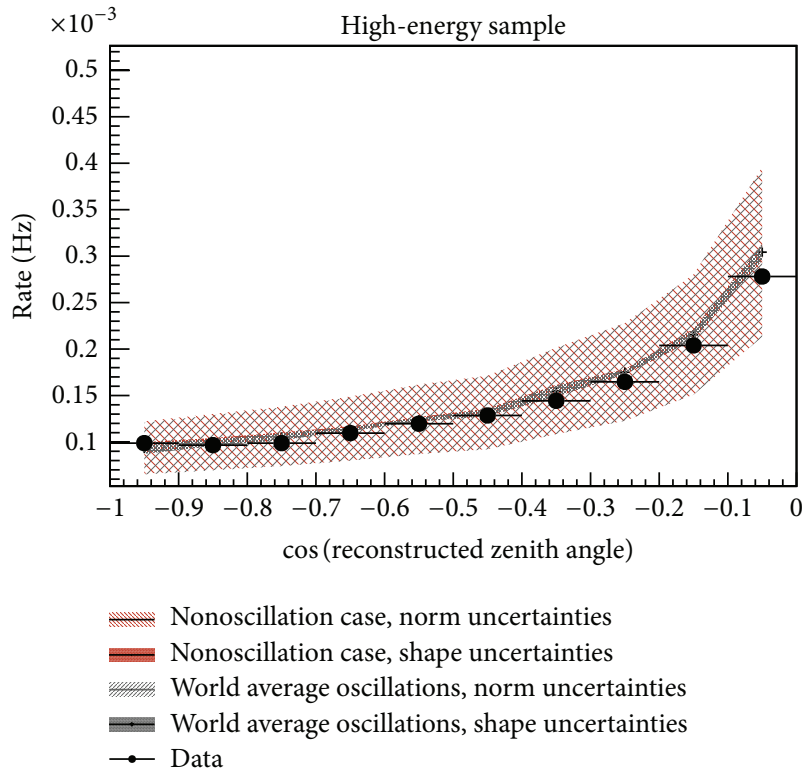

(b)

FIGURE 3: Data and Monte Carlo expectation at world average oscillation parameters [4] and at the nonoscillation scenario for the low-energy sample (a) and for the high-energy sample (b). The shaded bands illustrate separately those systematic effects which affect the normalisation (norm) and the shape (shape) of the distributions (figure from [25]).

IceCube data are under way. They have reported preliminary results recently.

6.2. IC79 Two-Dimensional Analysis. As this analysis [28] is well based on data in the IC79 configuration, here 312 days are used. The event selection requires that neutrino event candidates should have their interaction vertices inside the instrumented volume of the Deep-Core detector, which reduces background from high-energy neutrino events and downgoing atmospheric muons. When using the surrounding IceCube strings as a veto, their contributions can be partly discarded. Apart from the veto cut, only weak selection criteria are applied, which leads to a final event sample of 8117 events with an estimated purity in $\nu_{\mu}$ events of $70 \%$. The oscillation parameters are fitted on a two-dimensional distribution in zenith angle and energy. The zenith angle is reconstructed using standard IceCube methods [27]. The energy is derived from the measured track length [26]. For the likelihood, the standard Poisson formulation is used. For each bin $(i, j)$, the probability is calculated to observe $d_{i j}$ events in the measured data, given $s_{i j}$ events in the simulated data. In addition to fitting the oscillation parameters, nuisance parameters $q_{k}$ are also left free in the fit which absorb certain systematic uncertainties. Gaussian priors are used for each nuisance parameter $k$ with a mean value $\left\langle q_{k}\right\rangle$ and a width $\sigma_{k}$ which are added to the likelihood. The full likelihood expression has the form

$$
\begin{aligned}
-\mathrm{LLH}= & \sum_{i, j}\left(s_{i j}-d_{i j} \ln s_{i j}\right) \\
& +\frac{1}{2} \sum_{k}\left(\frac{q_{k}-\left\langle q_{k}\right\rangle}{\sigma_{k}}\right)^{2} .
\end{aligned}
$$

Other systematics, which are not directly implemented in the fit, are evaluated by separate simulations. As a preliminary result of this analysis, the nonoscillation hypothesis is rejected by a likelihood ratio corresponding to 5.1 standard deviations. The corresponding 90\% C.L. contour is also shown in Figure 5.

6.3. IC86 Analysis. The second new analysis [29] uses a complementary approach. A strict selection of events with clusters of essentially unscattered Cherenkov photon hits is applied, following the procedure which had previously been used for the ANTARES oscillation search [21]. The described hit selection and fitting procedure has been adapted to IceCube/DeepCore. A lower energy threshold and a better zenith angle distribution are observed compared to the performances of the above mentioned analyses. Also the dependence of the result on uncertainties in the description of the ice properties is reduced as scattered hits are largely discarded in this analysis. The neutrino energy for the $\nu_{\mu}$ CC sample is estimated from the sum of the fitted cascade energy at the neutrino vertex and the muon energy obtained from the measured muon range. Figure 4 shows the performance of the reconstructions used for the final event selection. A zenith angle resolution of 7 degrees, comparable with the kinematic angle between the neutrino, and the muon in the sample, is obtained. The energy is resolved with an error of 0.25 in $\log _{10}(E)$. The analysis was applied to the first year of data taken by the complete IceCube detector (IC86), from May 2011 until April 2012. In 343 days of lifetime, 1487 neutrino events were found. For the likelihood, the standard Poisson formulation with added nuisance terms is also used here (see (5)). The best fit values for single parameters obtained from the likelihood profile are $\sin ^{2}\left(2 \theta_{23}\right)=1$ (> 0.93 at $68 \%$ C.L. $)$ 

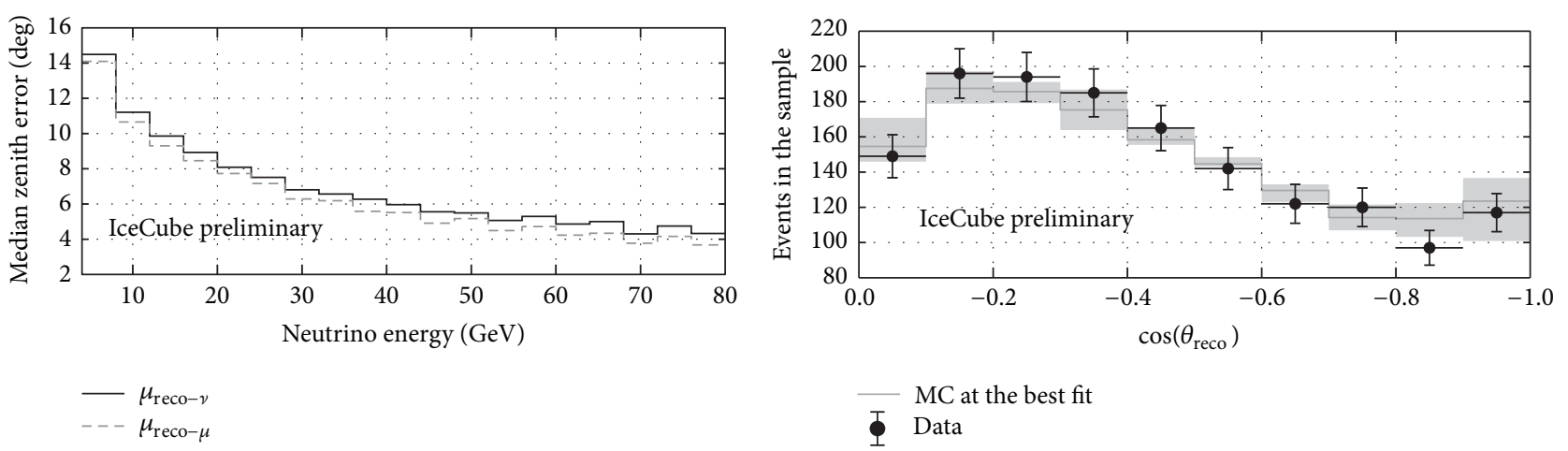

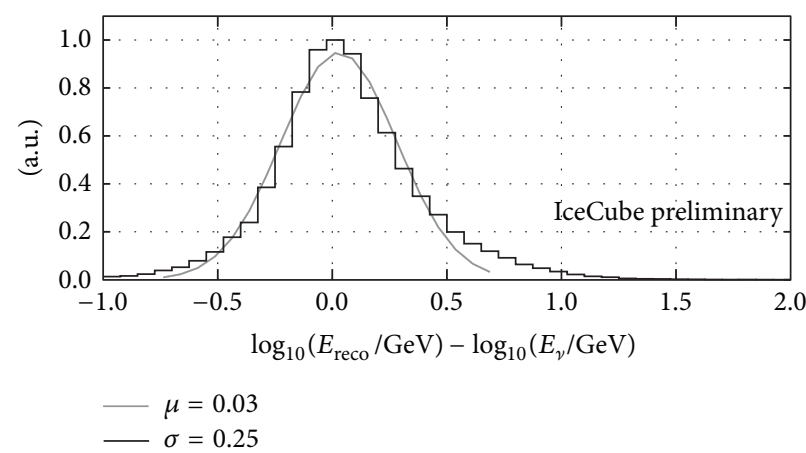

(a)

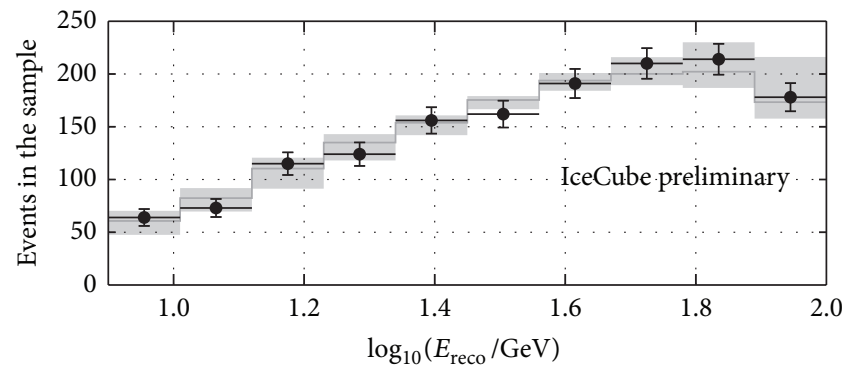

(b)

Figure 4: (a) Performance of reconstructions for the $v_{\mu}$ CC component of the IC86 analysis in zenith angle (top) and energy (bottom). (b) Zenith angle and energy distributions of data and best fit simulation. Statistical errors are added to the data; systematic errors are attributed to the simulation (figure from [29]).

and $\left|\Delta m_{32}^{2}\right|=(2.4 \pm 0.4) \cdot 10^{-3} \mathrm{eV}^{2}$. The nuisance parameters at the best fit point are all well within their one-sigma ranges. The resulting zenith angle and energy distributions for the best fit values are shown in Figure 4. The corresponding 90\% C.L. contour is also overlaid in Figure 5 and represents currently the best such measurement provided by a neutrino telescope.

Whereas the ANTARES oscillation measurement was based on four years of data, the current IceCube analyses did all use just one year of data. All of them are currently statistically limited. It can be expected that the measurement accuracy improves with roughly $\sqrt{T}$ ( $T$ being the detector lifetime) for a couple of years. But to reach a sensitivity, which would be competitive to the current MINOS [30] and SuperKamiokande [31] results, increased statistics alone are not sufficient. An improved energy estimator combined with a clean high-statistics low-energy event sample is needed. It has to be seen whether this can be accomplished within the DeepCore/IceCube setup or whether new, denser detectors are required.

\section{Projects for Low-Energy Extensions of Neutrino Telescopes}

All three mixing angles and both mass square differences of the neutrino mass eigenstates are known after the measurement of the mixing angle $\theta_{13}[5,6]$. Global fits of all experimental input [32] provide a coherent picture of the oscillation parameters. In the following, the best fit values from this analysis are used if not stated otherwise. One yet unknown feature of the oscillation scheme is the ordering of the mass eigenstates of the neutrinos, the neutrino mass hierarchy $(\mathrm{MH})$. Whereas $m_{2}>m_{1}$ is determined through matter effects in the sun, which influence the solar neutrino flux measurements, the options $m_{3}>m_{2}$ (normal hierarchy: $\mathrm{NH}$ ) and $m_{1}>m_{3}$ (inverted hierarchy: $\mathrm{IH}$ ) are both allowed. They could be distinguished by measuring matter effects in the Earth, which is considered to be in reach for the next generation of experiments. In the approximation of a constant electron density $n_{e}$ and for single $\Delta m_{32}^{2}$ dominance as derived for (3), its main effect is the modification of the mixing strength related to $\theta_{13}$, which has to be replaced by the effective matter mixing angle $\theta_{13}^{m}$ [33]:

$$
\sin 2 \theta_{13}^{m}=\frac{\sin \left(2 \theta_{13}\right)}{\sqrt{\left(2 \sqrt{2} G_{F} n_{e} E_{\gamma} / \Delta m_{32}^{2}-\cos 2 \theta_{13}\right)^{2}+\left(\sin 2 \theta_{13}\right)^{2}}}
$$

with $G_{F}$ the Fermi constant. The sign of $n_{e}$ is positive for neutrino interactions and negative for antineutrinos. A resonance, that is, $\sin 2 \theta_{13}^{m}=1$, occurs for neutrinos and $\Delta m_{32}^{2}>0(\mathrm{NH})$ and for antineutrinos and $\Delta m_{32}^{2}<0(\mathrm{IH})$. 


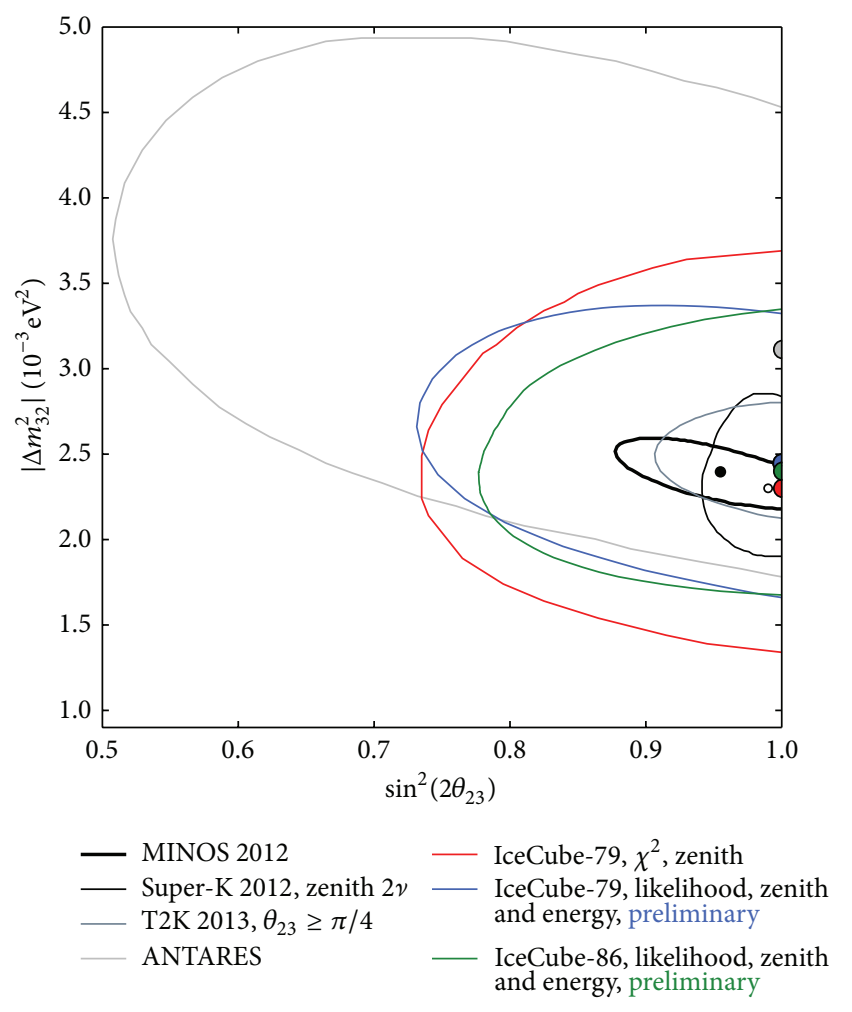

FIGURE 5: 90\% C.L. contours from recent measurements of the neutrino oscillation parameters by ANTARES [22] and IceCube $[25,28,29]$ compared to earlier measurements by MINOS [30] and Super-Kamiokande [31] (figure kindly provided by S. Euler, Aachen University).

For typical densities in the Earth mantle, the resonance is observed at $E_{\gamma}=6-8 \mathrm{GeV}$.

Neutrino oscillation probabilities are now calculated in a full three-flavour scheme including matter effects in the Earth. The Globes package $[34,35]$ is used for this purpose. Figure 6 shows the survival probabilities for upgoing atmospheric $\nu_{\mu}$ for different zenith angles as function of neutrino energy. The Earth density profile is taken from the PREM model [36]. The two colors refer to the two MH choices. A striking difference of the oscillation probabilities for the two options can be seen for zenith angles $\cos \Theta>0.4$ and energies close to the resonance energy of the matter effect in the Earth mantle. Neutrino interactions in this energy range can occur in various channels: quasielastic, via nuclear resonances or as deep-inelastic reactions. Moreover nuclear effects should be taken into account. Most of the studies mentioned below are based on the event generator GENIE [37] which comprises all mentioned effects.

It has been proposed to determine the $\mathrm{MH}$ by measuring atmospheric neutrinos [38] which has motivated proposals for low-energy extensions of existing and future neutrino telescope projects. To measure reliably neutrinos with energies of few GeVs, higher densities of the photosensor arrays are needed. Two such detectors have been proposed, which are presented in the following.
7.1. ORCA. KM3NeT [39] will be the next generation neutrino telescope in the Mediterranean Sea with an effective volume of several cubic kilometers. Funds for a first phase of the project, corresponding to about $20 \%$ of the total envisaged budget, are meanwhile available. A dedicated "Oscillation Research with Cosmics in the Abyss" (ORCA) is currently under evaluation as a low energy part of the KM3Net detector [40]. ORCA might consist of at least 50 detector lines, each equipped with 20 optical modules. Each optical module hosts 31 3-inch phototubes, as designed in the KM3NeT TDR [39]. Optical modules with many small phototubes have an enhanced photon counting capability and provide better directional information than larger tubes. This can be exploited in reconstruction algorithms and for background rejection. The readout will be largely simplified compared to ANTARES. For each detected pulse, only a timestamp and the "time over threshold" will be recorded. Trigger algorithms will be applied in an onshore computing farm, as successfully done for ANTARES. The distance of the detector lines on the sea bed will be approximately $20 \mathrm{~m}$, a value determined by deployment safety. The vertical distance between modules on a detector line will be chosen between $5 \mathrm{~m}$ and $10 \mathrm{~m}$, the precise value being subject to optimization. This leads to an equipped water mass of about 2 Mtons. A newly developed and simplified deployment scheme [39] will allow deploying several detector lines during one sea operation. It can be envisaged to complete the detector installation within 5 years.

Studies have been carried out, to evaluate the achievable resolution in zenith angle and energy. The reconstruction methods have been derived from ANTARES and have been adapted to the denser geometry of the ORCA detector. Preliminary results have been published recently [40] and they are shown in Figure 7. For events which interact inside the instrumented volume, a zenith angle resolution close to the kinematical limit between neutrino and muon direction can be reached. $68 \%$ of events with a muon of a true energy between 3 and $8 \mathrm{GeV}$ can be reconstructed with a precision of $3 \mathrm{GeV}$ from range estimation of the fitted track. An effective mass of 1.8 Mtons is observed for $\nu_{\mu}$ CC events with $E_{v}>5 \mathrm{GeV}$, having their interaction vertex inside the instrumented volume.

7.2. PINGU. PINGU stands for "Precision IceCube Next Generation Upgrade", and it is planned as a dense core extension of the existing IceCube/DeepCore ensemble [41]. Several geometries are under consideration. The two most promising configurations include additional 20-40 detector strings, each holding 60 to 120 light detectors, deployed within the DeepCore volume (see Figure 8 for the example of the 20-lines configuration). For the 20-lines configuration the dependence of the effective mass for a sample of $\nu_{\mu}$ CC events and a condition about a minimal number of optical modules hit is shown in Figure 8. Different reconstruction algorithms are currently tested for PINGU, some of them derived from IceCube tools (see above) and some others are under development specifically for PINGU. Currently a median in zenith angle resolution of 8-15 degrees is reported for neutrino energies between 2 and $20 \mathrm{GeV}$ whereas the 

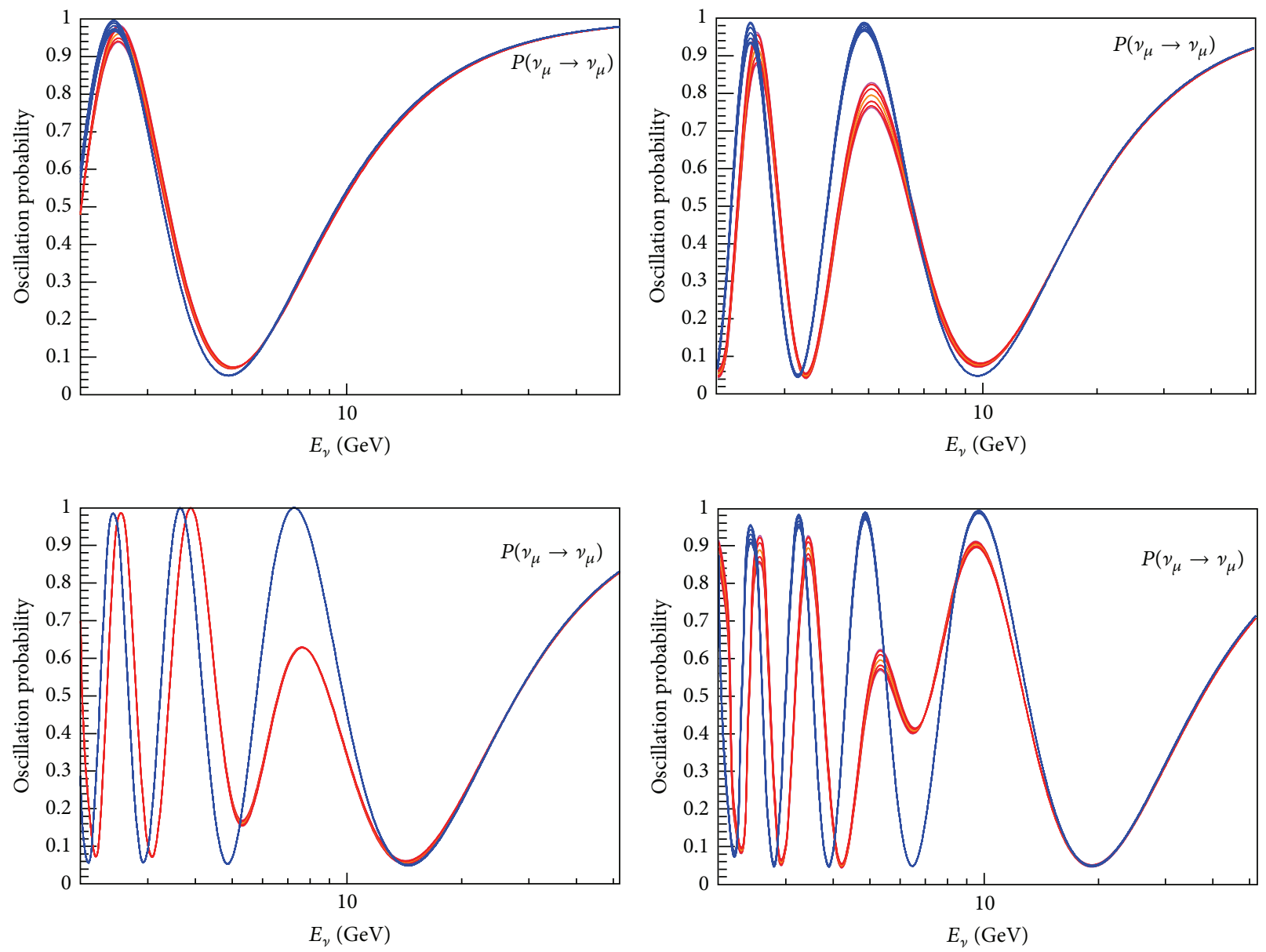

Figure 6: Neutrino oscillation probabilities $P\left(\nu_{\mu} \rightarrow v_{\mu}\right)$ as a function of neutrino energy for different zenith angles $\cos \Theta=0.2,0.4,0.6$, and 0.8 which correspond to baselines of $2548 ; 5097 ; 7645 ; 10194 \mathrm{~km}$. Oscillation parameters from a global fit are used. The group of red lines is for $\mathrm{NH}$, blue for $\mathrm{IH}$, and $\phi_{\mathrm{CP}}$ varied in steps of $30^{\circ}$ between $0^{\circ}$ and $330^{\circ}$.

median of the absolute neutrino energy resolution can be conveniently parametrised by $0.7 \mathrm{GeV}+0.2 \cdot E_{\nu}$ for an energy reconstruction which exploits the total brightness of fully contained events.

\subsection{Sensitivity for Neutrino Mass Hierarchy Determination} with Atmospheric Neutrinos. Despite the seemingly easy distinction of the two MH hypotheses for a given oscillation channel (as illustrated in Figure 6), the distinction of the two hierarchy hypotheses with atmospheric neutrinos is challenging due to the cancellation of contributions from neutrinos and antineutrinos. Further cancellation effects occur between $v_{e}$ and $v_{\mu}$ assuming an unavoidably imperfect flavour tagging method. The question of flavour tagging, that is, distinguishing cascade from track signatures and thereby isolating the $v_{\mu} \mathrm{CC}$, signal has still to be improvedin ORCA as well as in PINGU. The reachable sensitivity for $\mathrm{MH}$ is further attenuated by the finite energy and angular resolutions of the considered detectors which are discussed above. Last but not least $\mathrm{MH}$ effects can be partly masked by readjusting oscillation parameters (in particular $\Delta m_{32}^{2}$ and $\sin ^{2} 2 \theta_{23}$ ) within their present uncertainties.
Both ORCA and PINGU have yet to provide a sensitivity calculation for $\mathrm{MH}$, including all these effects plus detector and flux related sources of systematic uncertainties. The most detailed sensitivity study to date is based on input from PINGU performance figures and can be found in [42]. Its main result is shown in Figure 9. A $1.3 \sigma$ to $2.9 \sigma$ significance might be reached for three years running with the 20-lines PINGU detector depending on the true $\mathrm{MH}$ choice and the true values for other oscillation parameters. The result is based on a rather optimistic assumption of a cascade misidentification fraction of $5 \%$ and it assumes that the neutrino energy can be determined with a perfectly Gaussian resolution of $25 \%$.

The cancellation of matter effects between neutrinos and anti-neutrinos can be partly avoided by determining for each $v_{\mu}$ CC event the hadronic and muon part separately. This would allow to measure the inelasticity variable Bjorken$y$ which in turn could be used to statistically separate neutrinos from anti-neutrinos. The best separation would be reached for events of high inelasticity (large $y$ ), but these are hardly distinguishable from cascades due to the lowenergy muon tracks. The potential of the $y$ determination for the MH sensitivity is discussed in [43]. When assuming 


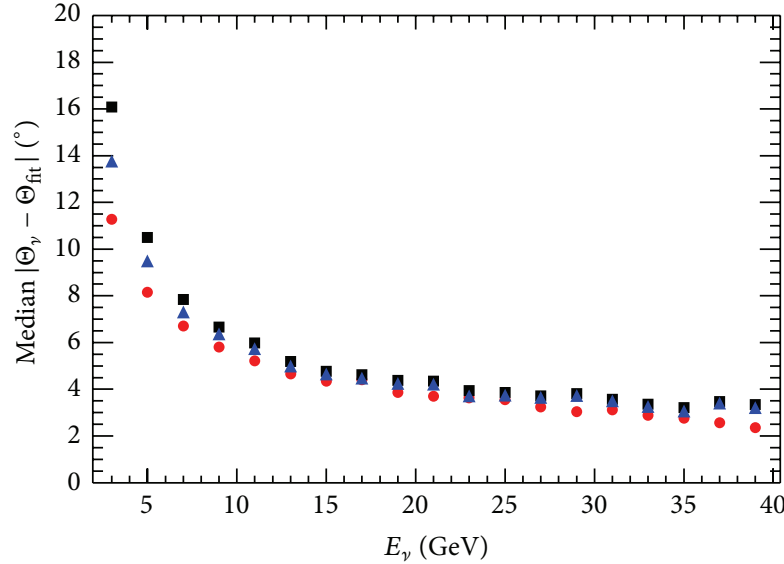

- Media $\Delta \Theta_{\gamma_{\text {gen }}+\mu_{\text {gen }}}$ (intrinsic angel)

- All reconstructed events

Events selected with a quality cut

(a)

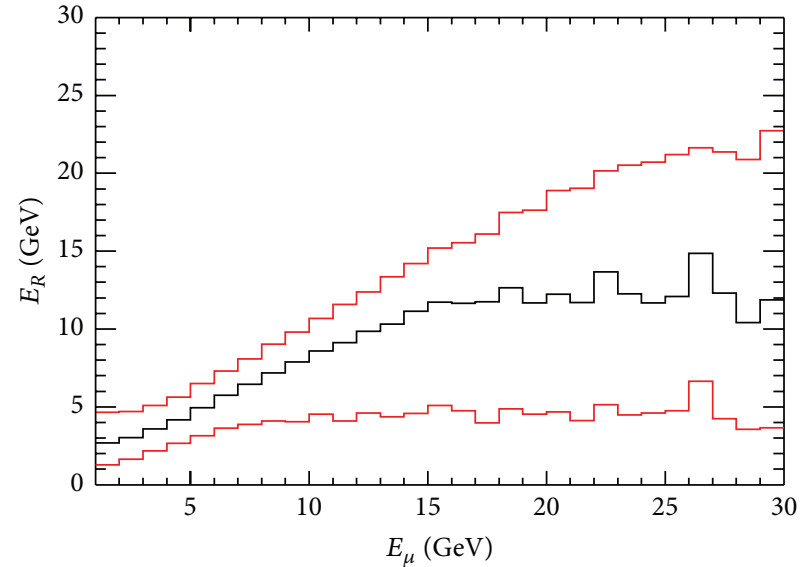

(b)

FIGURE 7: (a) Median of the difference between the true neutrino zenith angle and the reconstructed zenith angle calculated for all the upgoing reconstructed events with the interaction vertex inside the instrumented volume. For reference, the median of the difference between the true neutrino zenith angle and the true muon zenith angle is shown. (b) Reconstructed muon energy as a function of the muon true energy. The drawn black line gives the median value and the red lines indicate the $16 \%$ and $84 \%$ quantiles-the equivalent of a one-sigma range for a Gaussian distribution (figure from [40]).

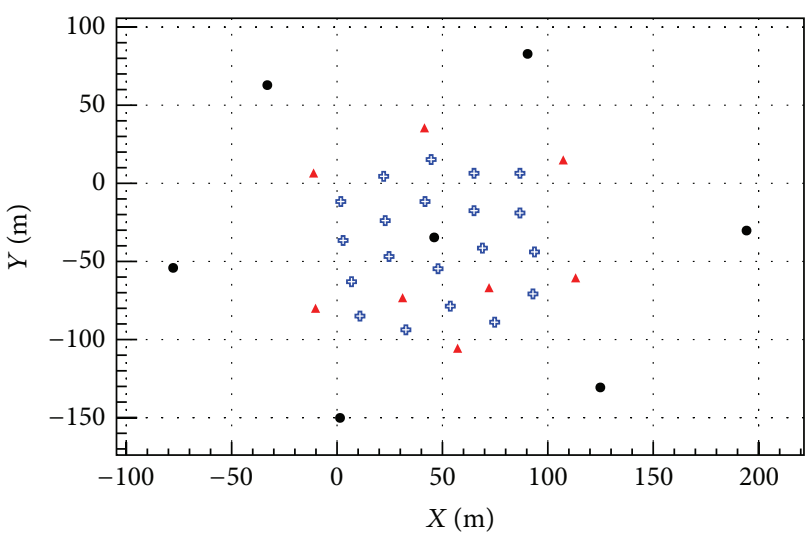

- IceCube

$\triangle$ DeepCore

$\Leftrightarrow$ PINGU

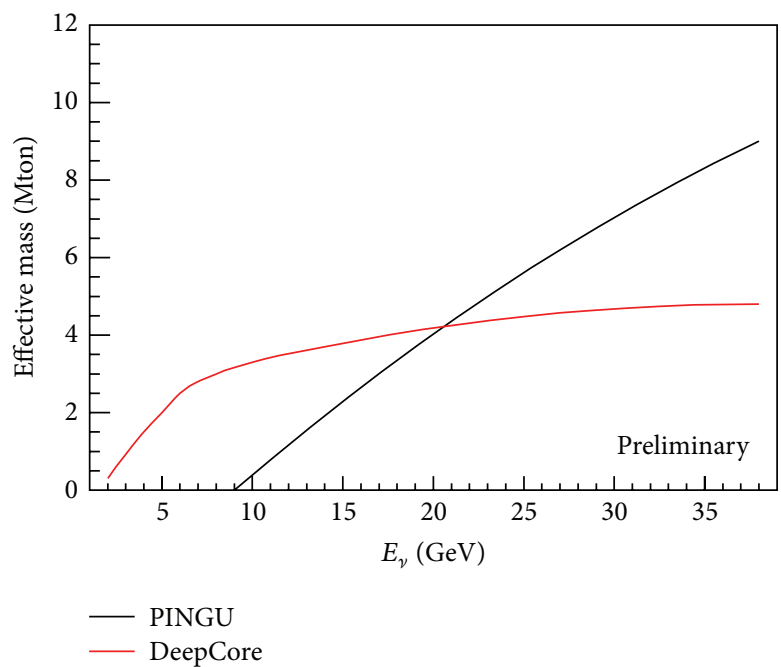

(b)

FIgURE 8: (a) Overhead view of the PINGU geometry layout with 20 additional detector strings. (b) Effective volume for PINGU in the baseline configuration (20 strings) in comparison with DeepCore. A fiducial volume defined by a cylinder with radius of $75 \mathrm{~m}$ and a height of $332 \mathrm{~m}$ is assumed. A threshold of 20 hits per event was assumed (figure from [41]).

a realistic experimental smearing of the measured quantities, an enhancement of $25 \%$ is quoted in [43] by measuring hadrons and muons separately.

The study of the neutrino mass hierarchy is not the only physics goal of PINGU and ORCA. The knowledge of the oscillation parameters $\Delta m_{32}^{2}$ and $\sin ^{2} \theta_{23}$ itself can be improved. The octant of the mixing angle can be determined, if its value is sufficiently distinct from maximal mixing and
$\left|\Delta m_{32}^{2}\right|$ can be measured with a precision better than $1 \%$ based on one year of data with PINGU alone [41] under the assumption that systematic effects such as the uncertainty on the ice model do not deteriorate this measurement too strongly. Further, indirect dark matter searches from annihilation processes of gravitationally confined particles can be extended toward lower masses compared to current results from ANTARES and IceCube. 


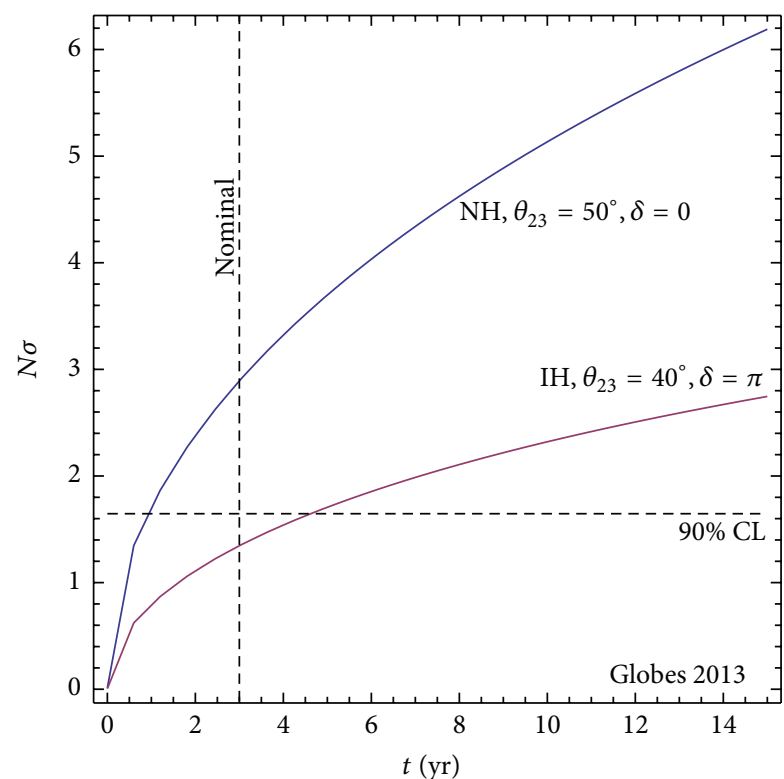

FIGURE 9: Number of $\sigma$ for the hierarchy discovery of a 20-lines PINGU detector as a function of time for two extreme cases of the true parameter values (figure from [42]).

\section{Neutrino Beams and Neutrino Telescopes}

The above-mentioned complications of determining $\mathrm{MH}$ with atmospheric neutrinos are avoided by counting beam related events of a specific flavour. Neutrino beams can be rather pure $\nu_{\mu}$ beams, that is, with low contamination from other flavours or polarities. Assuming a moderate capability of the target neutrino telescope to distinguish track signatures $\left(\nu_{\mu}\right.$ CC events or $\nu_{\tau}$ CC events with subsequent muonic decay) from cascade signatures (everything else), two basic methods have been proposed to determine $\mathrm{MH}$.

8.1. Muon Counting. Track signatures are isolated and beam related events are counted. This has been recently proposed [44] as a simple method to measure $\mathrm{MH}$ via the disappearance channel $P(\mu \rightarrow \mu)$. Assuming a conventional narrow band beam with a mean neutrino energy of $6-8 \mathrm{GeV}$, one can examine Figure 6 to determine the optimal baseline for such a measurement. The cleanest distinction of the two $\mathrm{MH}$ hypothesis is seen for $\cos \Theta=0.6$ or equivalently a baseline of $7645 \mathrm{~km}$. The ignorance about the CP-phase does not affect the distinction as one can immediately verify from Figure 6. Taking into account locations of existing particle accelerators and existing or potential future neutrino telescopes, a beamline from Fermilab to a KM3Net site close to Sicily is proposed [44] which yields a baseline of $7800 \mathrm{~km}$. For $10^{20}$ protons on target, about $1000 \nu_{\mu}$ CC events are expected in a Megaton detector such as ORCA for NH. This number is reduced by $30 \%$ for $\mathrm{IH}$, providing a $9 \sigma$ separation of the two hypotheses on purely statistical ground.

8.2. Electron Counting. An alternative approach exploits the appearance channel $P\left(\nu_{\mu} \rightarrow v_{e}\right)$ by counting cascadelike events [45]. Figure 10 shows the oscillation probability
$P\left(\nu_{\mu} \rightarrow v_{e}\right)$ for two very long baselines. The left plot corresponds to the distance Fermilab-Sicily (discussed above) the right plot is typical for a beamline from any accelerator in the Northern hemisphere to the South Pole, as discussed in [42]. In both cases, a clear separation of the hierarchy hypotheses is seen and it could be exploited in a counting experiment of cascade-like events. To find the optimal baseline for such a measurement, the dependence of the event rate $N$ from the baseline $L$ has to be taken into account: $N \propto L^{-2}$. For a typical neutrino beam, which provides neutrinos in the energy range $2-8 \mathrm{GeV}$, the event rate dependence from baseline is shown in Figure 11. An optimal separation between $\mathrm{NH}$ and $\mathrm{IH}$ is found for $\cos \Theta=0.2$ or equivalently a baseline of about $2600 \mathrm{~km}$. A beam with such a baseline could be built from the Institute of High Energy Physics (IHEP) in Protvino near Moscow (located at $54^{\circ} 52^{\prime} \mathrm{N}, 37^{\circ} 11^{\prime} \mathrm{E}$ [46]) towards one of the potential sites for the ORCA detector. The IHEP hosts the U70 proton accelerator [47] which provides protons with energies up to $70 \mathrm{GeV}$. It is operational since 1967 and it had been the world largest proton accelerator at its time of commissioning. For the calculation of event numbers below it is assumed that after an intensity upgrade, $N_{\text {pot }}=1.5 \cdot 10^{21}$ can be provided within few years, similar to what is planned for the NOVA experiment [48]. If the ORCA detector would be built close to the ANTARES site $\left(42^{\circ} 48^{\prime} \mathrm{N}, 6^{\circ} 10^{\prime} \mathrm{E}\right)$, a neutrino beam from Protvino to this location would result in a baseline of $2588 \mathrm{~km}$. An alternative site in the Ionian Sea off the Sicilian coast results incidentally in an identical baseline (within 1\%) for a beam from Protvino. A moderate downward inclination of $11.7^{\circ}$ is needed.

The corresponding oscillation probabilities $P\left(\nu_{\mu} \rightarrow\right.$ $\nu_{e}, v_{\mu}, v_{\tau}$ ) with the best fit parameters from [32] are shown in Figure 12. Most of the neutrino path will be in the outer Earth mantle with a maximal depth of $134 \mathrm{~km}$. A constant density of $3.3 \mathrm{~g} / \mathrm{cm}^{3}$ is used for the calculation of the oscillation probabilities as given in the PREM model [36] for the outer mantle.

For $P\left(\nu_{\mu} \rightarrow v_{e}\right)$, a significant difference between both hierarchies is observed in the range $3 \mathrm{GeV}<E_{v}<8 \mathrm{GeV}$. The variation of the CP-phase $\phi_{\mathrm{CP}}$ (different lines of the same color) leads instead only to moderate changes of the oscillation probabilities. Peak values of $11 \%$ for $P\left(\nu_{\mu} \rightarrow \nu_{e}\right)$ are observed in NH. This is smaller than that for larger baselines (see Figure 10), but fully compensated by the higher flux due to the smaller $L$ (see Figure 11). $P\left(\nu_{\mu} \rightarrow v_{\mu}\right)$ depends only weakly on $\mathrm{MH}$ and not on $\phi_{\mathrm{CP}}$. The first vacuum oscillation maximum at $5 \mathrm{GeV}$ is the dominating feature in the shown energy range. Counting $\nu_{\mu}$ events can serve as a flux normalisation or it could be used to improve the measurement of the atmospheric oscillation parameters $\left|\Delta m_{32}^{2}\right|$ and $\theta_{23}$.

To calculate event numbers, simplified cross-section formulas are used. The total cross sections for $\nu_{\mu}$ and $\bar{\nu}_{\mu}$ CC interactions are taken from [4] in the parton scaling approximation:

$$
\begin{aligned}
& \sigma_{\gamma_{\mu}}^{\mathrm{CC}}\left(E_{\nu}\right)=0.68 \cdot\left(E_{\nu} / \mathrm{GeV}\right) 10^{-38} \mathrm{~cm}^{2} \\
& \sigma_{\bar{\nu}_{\mu}}^{\mathrm{CC}}\left(E_{\nu}\right)=0.34 \cdot\left(E_{\nu} / \mathrm{GeV}\right) 10^{-38} \mathrm{~cm}^{2} .
\end{aligned}
$$




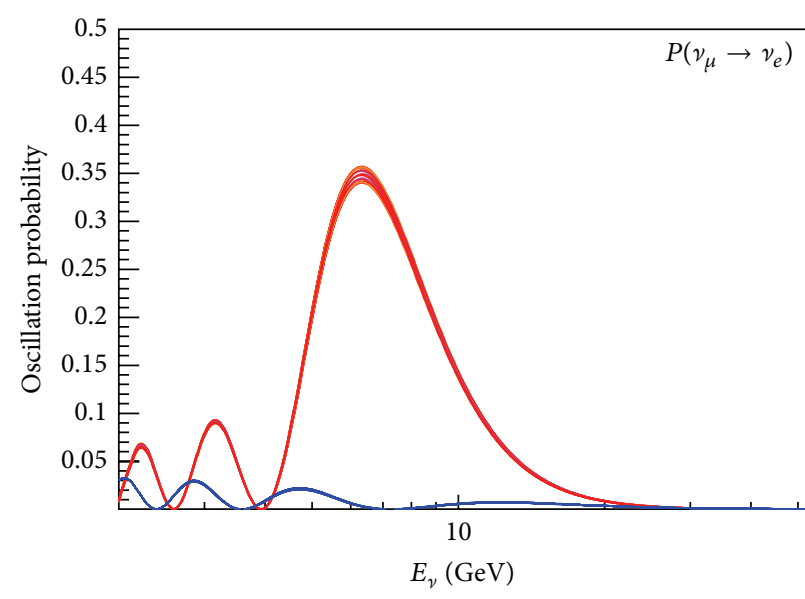

(a)

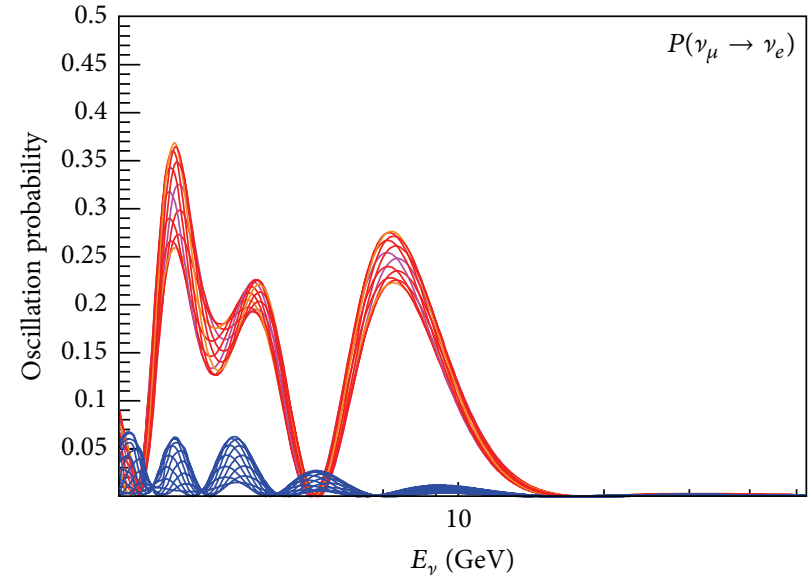

(b)

Figure 10: Neutrino oscillation probabilities $P\left(\nu_{\mu} \rightarrow \nu_{e}\right)$ parameters from a global fit as a function of neutrino energy for baselines of $7675 \mathrm{~km}$ (a) and $11468 \mathrm{~km}$ (b) which corresponds to $\cos \Theta=0.6$ and 0.9 , respectively. The group of red lines is for $\mathrm{NH}$, blue for $\mathrm{IH}$ and $\phi_{\mathrm{CP}}$ varied in steps of $30^{\circ}$ between $0^{\circ}$ and $330^{\circ}$.

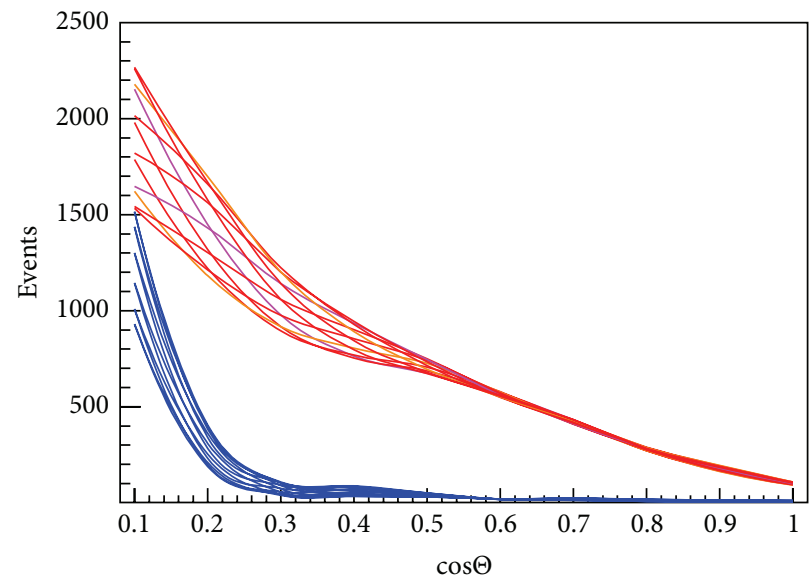

(a)

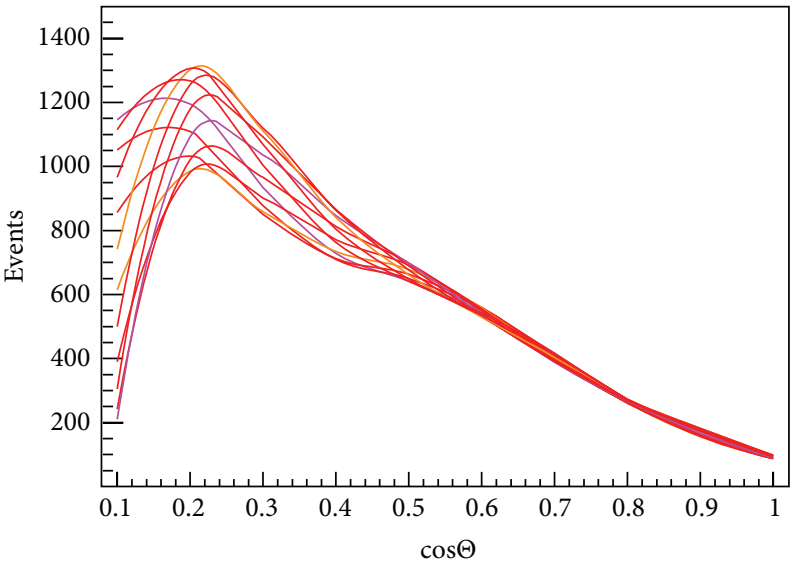

(b)

FIGURE 11: Event rates of oscillated $v_{e}$-CC events as function of $\cos \Theta$, which is proportional to the baseline. (a) Comparison of event rates for both hierarchy hypotheses (same color code as in earlier figures). (b) Event rate differences from left plot.

Deviations from this linear behaviour due to quasielastic or resonant interactions are ignored. Their contribution would not alter the result of this study in a significant way. The relevant neutrino energies are significantly larger than $m_{\mu}$ and $m_{e}$; therefore, from flavour universality, $\sigma_{v_{e}}^{\mathrm{CC}}=\sigma_{v_{\mu}}^{\mathrm{CC}}$ and $\sigma_{\bar{v}_{e}}^{\mathrm{CC}}=\sigma_{\bar{v}_{\mu}}^{\mathrm{CC}}$. However for $\nu_{\tau}$ CC interactions, the mass of the $\tau$ lepton cannot be neglected. We use the calculation from [49]. For a threshold energy $E_{0}=5 \mathrm{GeV}$ and $E_{0}<E_{v}<30 \mathrm{GeV}$, we find the following simple parametrisation:

$$
\sigma_{\nu_{\tau}}^{\mathrm{CC}}\left(E_{\nu}\right)=0.29 \log \left(\frac{E_{\nu}}{E_{0}}\right) \sigma_{v_{\mu}}^{\mathrm{CC}}\left(E_{\nu}\right) .
$$

The cross sections for $v(\bar{v}) \mathrm{NC}$ interactions for all flavours are approximated as $1 / 3 \sigma_{v_{e}}^{\mathrm{CC}}\left(E_{\gamma}\right)$ and $1 / 3 \sigma_{\bar{v}_{e}}^{\mathrm{CC}}\left(E_{\nu}\right)$, respectively.
As a first step, the impact of the MH on the total event count is evaluated, regardless of its topology in the detector. Whereas the sum of the oscillation probabilities $\sum_{\alpha} P(\mu \rightarrow$ $\alpha$ ) equals unity due to the unitarity of the flavour mixing matrix, the cross section weighted sum

$$
P_{\mu}^{\sigma}\left(E_{\nu}\right)=\frac{\sigma_{\nu_{\mu}}^{\mathrm{NC}}\left(E_{\nu}\right)+\sum_{\alpha} P(\mu \longrightarrow \alpha) \sigma_{\nu_{\alpha}}^{\mathrm{CC}}\left(E_{\nu}\right)}{\sigma_{\nu_{\mu}}^{\mathrm{NC}}\left(E_{0}\right)+\sigma_{\nu}^{\mathrm{CC}}\left(E_{0}\right)}
$$

(arbitrarily normalised at $E_{0}=1 \mathrm{GeV}$ ) may help identifying the optimal energy range to separate the two $\mathrm{MH}$ hypotheses. $P_{\mu}^{\sigma}$ is shown on the left plot of Figure 13 and it can be interpreted as the event rate per neutrino energy seen in a detector with an energy independent detection efficiency for a pure $v_{\mu}$ flux flat in energy. No distinction is 


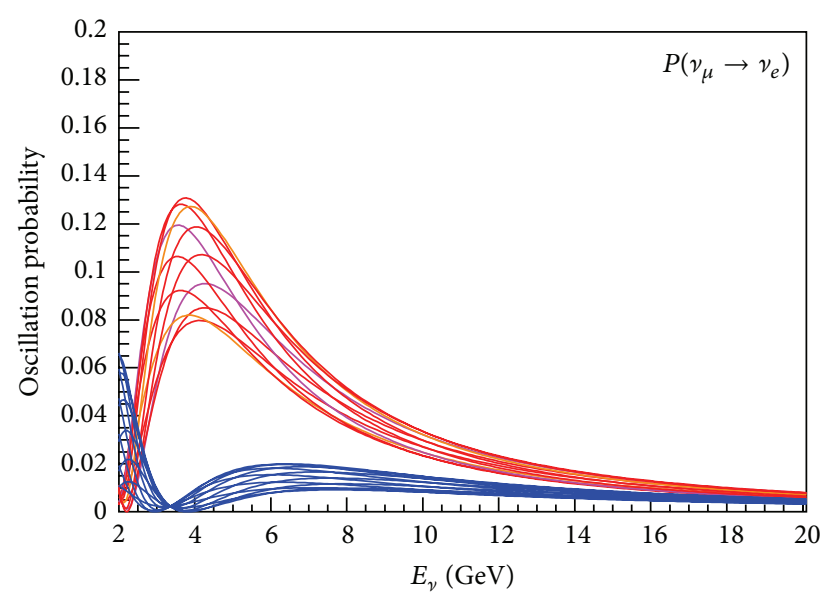

(a)

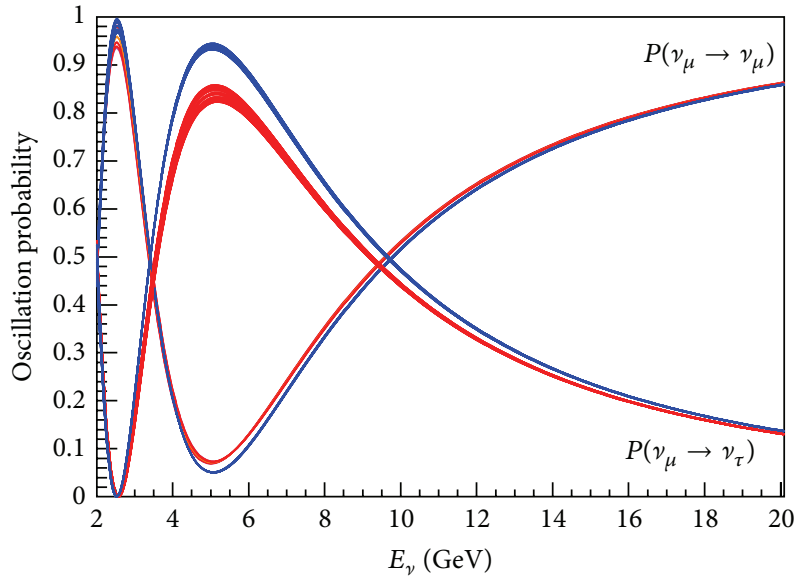

(b)

Figure 12: Neutrino oscillation probabilities $P\left(\nu_{\mu} \rightarrow v_{e}\right), P\left(v_{\mu} \rightarrow v_{\mu}\right)$ and $P\left(\nu_{\mu} \rightarrow v_{\tau}\right)$ for a baseline of $2600 \mathrm{~km}$ and oscillation parameters from a global fit as a function of neutrino energy. The group of red lines is for $\mathrm{NH}$, blue for $\mathrm{IH}$ and $\phi_{\mathrm{CP}}$ varied in steps of $30^{\circ}$ between $0^{\circ}$ and $330^{\circ}$. The special values $\phi_{\mathrm{CP}}=0^{\circ}, 180^{\circ}$ (CP conservation) are indicated in magenta, $\phi_{\mathrm{CP}}=90^{\circ}, 270^{\circ}$ (maximal CP violation) in orange on the left plot.

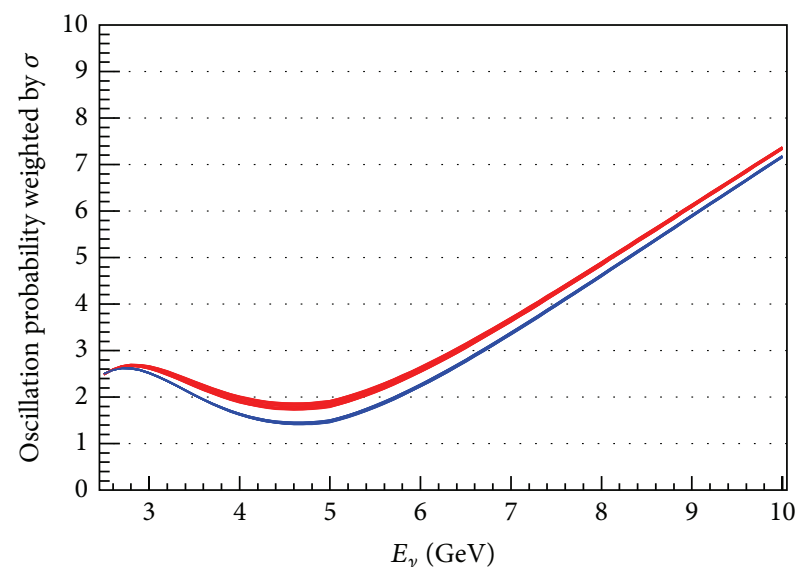

(a)

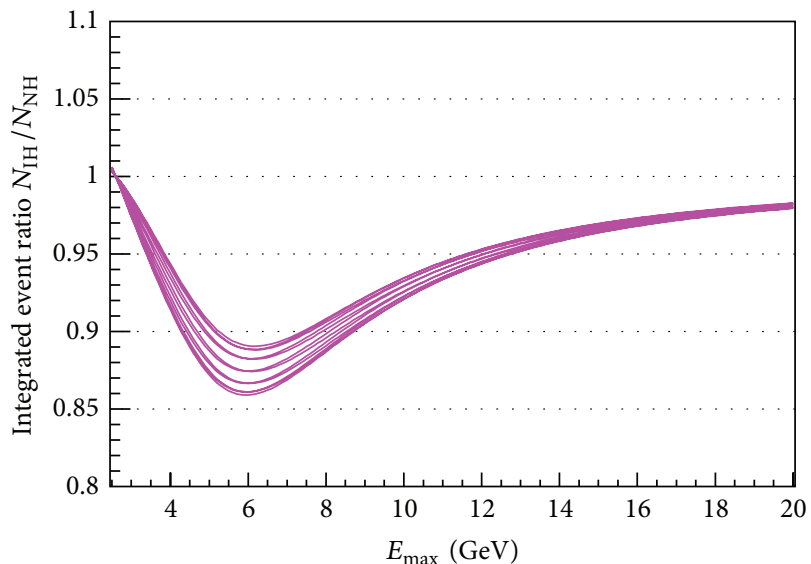

(b)

Figure 13: (a) Summed oscillation probabilities $P(\mu \rightarrow \alpha)$ weighted by cross-sections (red: NH, blue: IH). (b) Ratio of integrals $N_{\text {IH }} / N_{\text {NH }}$ from left plot between $E_{\min }=2.5 \mathrm{GeV}$ and $E_{\max }$.

made neither between NC and CC interactions nor between different flavours. Nevertheless, a clear separation of the two $\mathrm{MH}$ hypotheses is observed for energies above $3 \mathrm{GeV}$. It can be attributed to the kinematical suppression of $\nu_{\tau} \mathrm{CC}$ interactions. The size of the effect is quantified in the right plot of Figure 13 which shows the ratio between $\mathrm{IH}$ and $\mathrm{NH}$ integrals from the left plot (for different values of $\phi_{\mathrm{CP}}$ ) taken between an assumed threshold energy of $2.5 \mathrm{GeV}$ and a variable maximal energy $E_{\max }$. An optimal value of $E_{\max }=$ $6 \mathrm{GeV}$ can be read from the figure, which yields a suppression of the IH event rate of 11-14\% compared to the expected rate for $\mathrm{NH}$. Extending the energy range to higher values reduces the relative size of the separation of the $\mathrm{MH}$ hypotheses. It can be concluded that a measurement of the mass hierarchy will be possible even without any flavour tagging capabilities using a neutrino beam in a limited energy range of 2$6 \mathrm{GeV}$ and a large detector which can reliably count beam related neutrino interactions. Nevertheless, flavour tagging methods are discussed below and they are used to improve the significance of the measurement.

In the past, a neutrino beam was provided to several experiments, among them the bubble chamber SKAT [47]. Secondary hadrons were produced in an aluminum target and focused by parabolic lenses. Neutrinos were produced in a $140 \mathrm{~m}$ long decay line. The bubble chamber was situated $270 \mathrm{~m}$ behind the target and at a distance of $l_{\text {SKAT }}=245 \mathrm{~m}$ downstream the beginning of the decay tube. This value will be used in the following to scale the beam intensity to the remote location. The neutrino fluxes $d \Phi_{v} / d E_{v}$, as they were delivered to the SKAT experiment for focusing of positively 


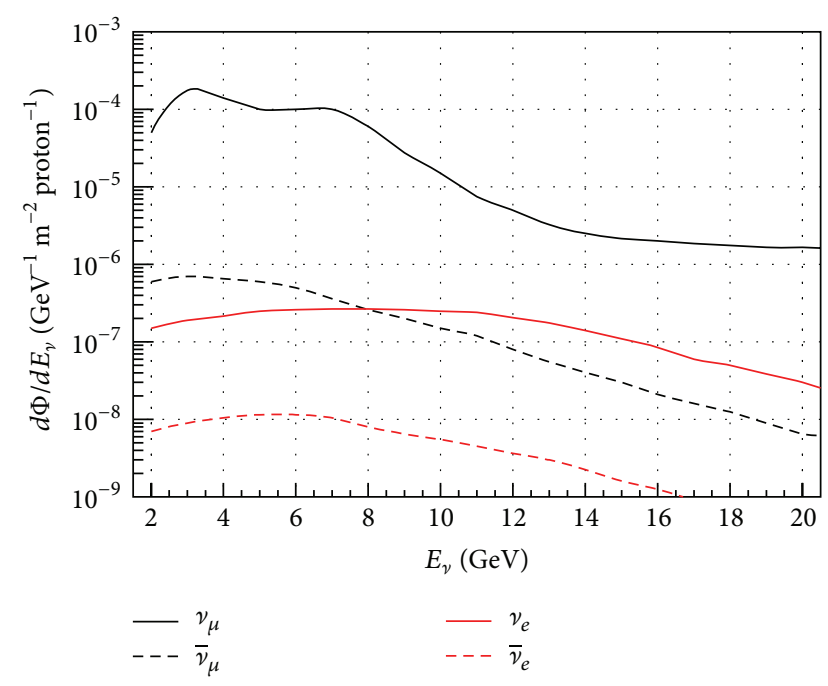

FIGURE 14: Neutrino flux $d \Phi_{\gamma} / d E_{\gamma}$ per proton as seen by the SKAT bubble chamber. The different lines correspond to the different neutrino flavours.

charged hadrons and for all four flavours present in the beam, are shown in Figure 14. A parametrisation of the beam intensity as function of the neutrino energy at the SKAT detector has been obtained from $[47,50]$ and it will be used in the following. As seen from Figure 14, a very clean $\nu_{\mu}$ beam was provided with energies dominantly in the range $2-8 \mathrm{GeV}$. Contaminations from other flavours were on the subpercent level.

The rate of detected CC events of flavour $\alpha$ can now be calculated as follows:

$$
\begin{aligned}
& \frac{d N_{\alpha}}{d E_{\nu}}= N_{\text {pot }}\left(\frac{l_{\mathrm{SKAT}}}{l_{\mathrm{LBL}}}\right)^{2} \frac{M_{\mathrm{eff}}\left(E_{\nu}\right)}{m_{p}} \\
& \times\left[\sigma_{v_{\alpha}}^{\mathrm{CC}}\left(\frac{d \Phi_{\nu_{\mu}}}{d E_{\nu}} P_{\mu \alpha}+\frac{d \Phi_{v_{e}}}{d E_{\nu}} P_{e \alpha}\right)\right. \\
&\left.+\sigma_{\bar{v}_{\alpha}}^{\mathrm{CC}}\left(\frac{d \Phi_{\bar{\nu}_{\mu}}}{d E_{\nu}} P_{\overline{\mu \alpha}}+\frac{d \Phi_{\bar{\nu}_{e}}}{d E_{\nu}} P_{\overline{e \alpha}}\right)\right]
\end{aligned}
$$

with $m_{p}$ the proton mass and the abbreviation $P_{\beta \alpha}=P\left(v_{\beta} \rightarrow\right.$ $\left.v_{\alpha}\right)$ and $P_{\overline{\beta \alpha}}=P\left(\bar{v}_{\beta} \rightarrow \bar{v}_{\alpha}\right)$ for the oscillation probabilities. The effective mass $M_{\text {eff }}\left(E_{\gamma}\right)$ is taken from Section 7.1 and it is assumed to be flavour independent. This overestimates the relative $v_{\tau}$ CC detection efficiency due to the escaping neutrino(s) of the tau decay. As $\nu_{\tau}$ interactions are a background in the present analysis, this is a conservative approximation. For $N_{\text {pot }}=1.5 \cdot 10^{21}$, one obtains $d N_{e} / d E_{v}=1621 \pm 255$ for $\mathrm{NH}$ but only $d N_{e} / d E_{v}=497 \pm 100$ for IH. The errors correspond to the uncertainty of the CP-phase. The statistical separation of both samples is better than $20 \sigma . d N_{\mu} / d E_{v}$ depends only weakly on MH (10317 versus 10015) and it can serve as reference sample.

The last step consists in adding background channels and considering misidentification for the two topologies. Contributions from atmospheric neutrinos and misreconstructed downgoing atmospheric muons can be ignored. A pulsed beam with a typical duty cycle lower than $10^{-6}$ allows to safely discard these events. The beam itself is a source of background events. $\nu_{\tau}$ CC events with a muonic $\tau$-decay produce "track" events, whereas $\mathrm{NC}$ events and $\nu_{\tau}$ CC events with a nonmuonic $\tau$-decay have a genuine cascade signature. The rate of detected NC events is given by

$$
\begin{aligned}
\frac{d N_{\mathrm{NC}}}{d E_{\nu}}= & N_{\mathrm{pot}}\left(\frac{l_{\mathrm{SKAT}}}{l_{\mathrm{LBL}}}\right)^{2} \frac{M_{\mathrm{eff}}\left(E_{\nu} / 2\right)}{m_{p}} \\
\times & {\left[\sigma_{\nu}^{\mathrm{NC}}\left(\frac{d \Phi_{\nu_{\mu}}}{d E_{\nu}}+\frac{d \Phi_{v_{e}}}{d E_{\nu}}\right)\right.} \\
& \left.+\sigma_{\bar{v}}^{\mathrm{NC}}\left(\frac{d \Phi_{\bar{\gamma}_{\mu}}}{d E_{\nu}}+\frac{d \Phi_{\bar{\nu}_{e}}}{d E_{\nu}}\right)\right] .
\end{aligned}
$$

No dependence on oscillation parameters enters here. As, on average, $50 \%$ of the neutrino energy is transferred to the outgoing neutrino, the effective mass $M_{\text {eff }}$ is evaluated at $E_{\gamma} / 2$.

In addition, the probability $\epsilon\left(E_{\gamma}\right)$ to misidentify a cascade as track is introduced, while the misidentification of a track as cascade will be called $\eta\left(E_{\nu}\right)$. Both functions are parametrised by

$$
\epsilon\left(E_{\nu}\right)=\eta\left(E_{v}\right)=\frac{1}{\left(E_{\nu} / \mathrm{GeV}\right)} ; \quad E_{v}>2 \mathrm{GeV}
$$

For $E_{v}=2 \mathrm{GeV}, \epsilon=\eta=0.5$, which means that the two topologies cannot be distinguished and the attribution of an event to one of them is random. A $5 \mathrm{GeV}$ neutrino produces a muon with an average range of $15 \mathrm{~m}$ in sea water which exceeds already by far the typical longitudinal size of a hadronic or electromagnetic shower. Correspondingly, the misidentification probability is assumed to drop to $20 \%$. For $E_{v}=10 \mathrm{GeV}, \epsilon$ and $\eta$ further decrease to $10 \%$. With these two quantities, the total background for the two event samples can be written as

$$
\begin{aligned}
\frac{d N_{\mathrm{bg}}^{\mathrm{track}}}{d E_{\nu}}= & \epsilon \frac{d N_{\mathrm{sig}}^{\mathrm{casc}}}{d E_{v}} \\
& +\left[\epsilon\left(1-\mathrm{BR}_{\tau \mu}\right)+(1-\eta) \mathrm{BR}_{\tau \mu}\right] \\
& \times \frac{d N_{\tau}}{d E_{\nu}}+\epsilon \frac{d N_{\mathrm{NC}}}{d E_{\nu}} \\
\frac{d N_{\mathrm{bg}}^{\text {casc }}}{d E_{\nu}}= & \eta \frac{d N_{\mathrm{sig}}^{\text {track }}}{d E_{\nu}} \\
& +\left[(1-\epsilon)\left(1-\mathrm{BR}_{\tau \mu}\right)+\eta \mathrm{BR}_{\tau \mu}\right] \\
& \times \frac{d N_{\tau}}{d E_{v}}+(1-\epsilon) \frac{d N_{\mathrm{NC}}}{d E_{\nu}} .
\end{aligned}
$$

$\mathrm{BR}_{\tau \mu}$ stands for the muonic branching ratio of the tau decay (17.4\%). The total number of observed events in each channel 


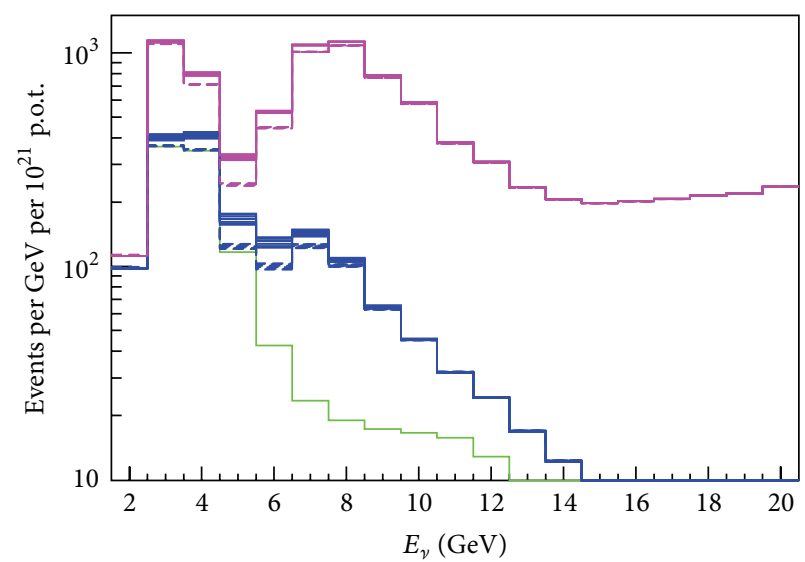

(a)

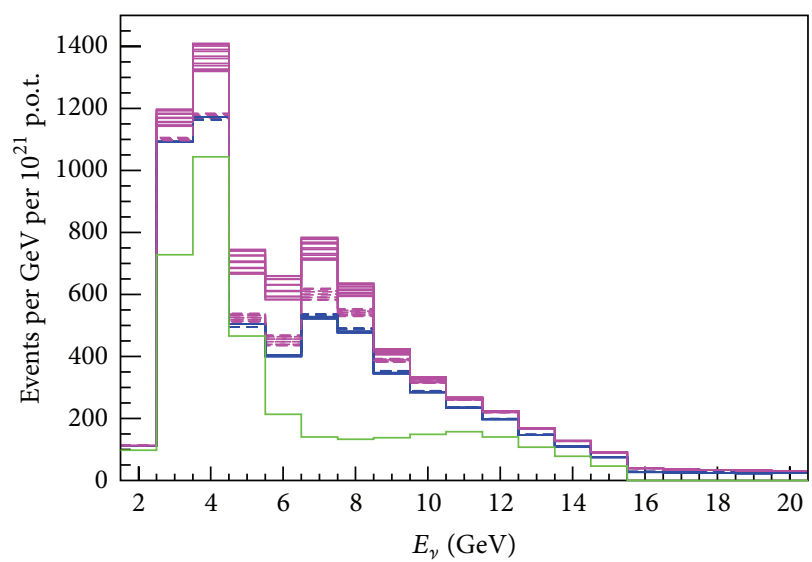

(b)

FiguRE 15: Observed events for $N_{\text {pot }}=1.5 \cdot 10^{21}$ as function of the neutrino energy. (a) Track-like events. (b) Cascade-Like events. In green the background from NC events is given. The blue histogram shows the total background including misreconstructed CC events. Magenta histograms are for the total event rate (signal plus background) for $\mathrm{NH}$ (solid) and $\mathrm{IH}$ (dashed) and various values of $\phi_{\mathrm{CP}}$.

TABLE 1: Event numbers for $N_{\text {pot }}=1.5 \cdot 10^{21}$ in the track and cascade channel for both mass hierarchy schemes and varying $\phi_{\mathrm{CP}}$ values.

\begin{tabular}{|c|c|c|c|c|}
\hline Channel & Tracks NH & Tracks IH & Cascades NH & Cascades IH \\
\hline No oscil & \multicolumn{2}{|c|}{26315} & \multicolumn{2}{|c|}{-} \\
\hline Signal & 8990 & 8735 & $1134-1547$ & $350-519$ \\
\hline Misreco & $232-329$ & $47-79$ & 1326 & 1280 \\
\hline$\nu_{\tau}$ & $324-332$ & $351-355$ & $978-998$ & 1057-1068 \\
\hline $\mathrm{NC}$ & 1092 & 1092 & 3640 & 3640 \\
\hline BG Total & 1655-1745 & $1494-1522$ & 5944-5964 & 5977-5988 \\
\hline Total & $10645-10736$ & $10229-10257$ & $7099-7491$ & $6338-6496$ \\
\hline
\end{tabular}

is given by adding the (reduced) number of signal events and the background contribution from (13) as follows:

$$
\begin{aligned}
& \frac{d N_{\mathrm{tot}}^{\mathrm{track}}}{d E_{v}}=(1-\eta) \frac{d N_{\mathrm{sig}}^{\mathrm{track}}}{d E_{v}}+\frac{d N_{\mathrm{bg}}^{\mathrm{track}}}{d E_{v}} \\
& \frac{d N_{\mathrm{tot}}^{\mathrm{casc}}}{d E_{v}}=(1-\epsilon) \frac{d N_{\mathrm{sig}}^{\mathrm{casc}}}{d E_{v}}+\frac{d N_{\mathrm{bg}}^{\mathrm{casc}}}{d E_{v}} .
\end{aligned}
$$

The resulting rates are shown in Figure 15 and quoted in Table 1. The event rates between the two $\mathrm{MH}$ hypotheses differ by $9-18 \%$ with a statistical uncertainty of $1.2 \%$. The statistical significance of the MH hypothesis test is still better than $7 \sigma$ and it remains at the level of $3 \sigma$ even when adding an additional systematic uncertainty of 3-4\% (depending on the true value of the CP-phase) for the determination of the total cascade event rate. Figure 15 illustrates the total event numbers as function of neutrino energy, detailing the different contributions. NC events are added to the $E_{\gamma} / 2$ bins according to their lower light yield in the detector. Their main contribution is found for energies below $6 \mathrm{GeV}$. Background from CC events is instead mainly seen above $5 \mathrm{GeV}$. The separation of the $\mathrm{MH}$ hypotheses is most pronounced in the range $4-8 \mathrm{GeV}$.

\section{Conclusion}

Neutrino telescopes have been designed to identify cosmic sources of high-energy neutrinos. Both ANTARES and IceCube have demonstrated their capability to contribute as well to the measurement of neutrino oscillation parameters. With several years of IceCube/DeepCore sensitivities comparable to MINOS might be reachable. Low-energy extensions such as ORCA and PINGU will allow to lower the effective detection threshold to values of few $\mathrm{GeV}$. This will open the possibility to perform competitive measurements of neutrino oscillation parameters and to pin down yet unknown parameters such as the neutrino mass hierarchy or the octant of the mixing angle $\theta_{23}$. The significance of these future measurements with atmospheric neutrinos could be complemented by using accelerator neutrinos from yet to be built long-baseline beams pointing towards one of the future neutrino telescopes.

\section{References}

[1] F. Halzen and S. R. Klein, "Invited review article: IceCube: an instrument for neutrino astronomy," Review of Scientific Instruments, vol. 81, Article ID 081101, 2010.

[2] M. Ageron, J. A. Aguilar, I. Al Samarai et al., "ANTARES: the first undersea neutrino telescope," Nuclear Instruments and Methods in Physics A, vol. 656, pp. 11-38, 2011. 
[3] R. Abbasiab, Y. Abdouv, and T. Abu-Zayyadag, "The design and performance of IceCube DeepCore," Astroparticle Physics, vol. 35, no. 10, pp. 615-624, 2012.

[4] J. Behringer, J. F. Arguin, R. M. Barnett et al., "Review of particle physics," Physical Review D, vol. 86, no. 1, Article ID 010001, 1528 pages, 2012.

[5] F. P. An, J. Z. Bai, A. B. Balantekin et al., "Observation of electron-antineutrino disappearance at Daya Bay," Physical Review Letters, vol. 108, no. 17, Article ID 171803, 7 pages, 2012.

[6] J. K. Ahn, S. Chebotaryovet, J. H. Choi et al., "Observation of reactor electron antineutrino disappearance in the RENO experiment," Physical Review Letters, vol. 108, no. 19, Article ID 191802, 6 pages, 2012.

[7] S. Adrian-Martinez, M. Ageron, J. A. Aguilar et al., "The positioning system of the ANTARES neutrino telescope," Journal of Instrumentation, vol. 7, Article ID T08002, 2012.

[8] P. Amram, M. Anghinolfi, S. Anvaret et al., "The ANTARES optical module," Nuclear Instruments and Methods in Physics Research A, vol. 484, pp. 369-383, 2002.

[9] M. Ageron, J. A. Aguilar, A. Albert et al., "The ANTARES optical beacon system," Nuclear Instruments and Methods in Physics Research A, vol. 578, no. 3, pp. 498-509, 2007.

[10] J. A. Aguilar, I. Al Samarai, A. Albert et al., "Performance of the front-end electronics of the ANTARES neutrino telescope," Nuclear Instruments and Methods in Physics Research A, vol. 622, no. 1, pp. 59-73, 2010.

[11] J. A. Aguilar, I. Al Samarai, A. Albert et al., "Time calibration of the ANTARES neutrino telescope," Astroparticle Physics, vol. 34, no. 7, pp. 539-549, 2011.

[12] J. A. Aguilar, A. Albert, F. Ameli et al., "Study of large hemispherical photomultiplier tubes for the ANTARES neutrino telescope," Nuclear Instruments and Methods in Physics Research A, vol. 555, no. 1-2, pp. 132-141, 2005.

[13] J. A. Aguilar, A. Albert, F. Amelie et al., "The data acquisition system for the ANTARES neutrino telescope," Nuclear Instruments and Methods in Physics Research A, vol. 570, no. 1, pp. 107-116, 2007.

[14] G. Carminati, M. Bazzotti, A. Margiotta, and M. Spurio, "Atmospheric MUons from PArametric formulas: a fast GEnerator for neutrino telescopes (MUPAGE)," Computer Physics Communications, vol. 179, no. 12, pp. 915-923, 2008.

[15] Y. Becherini, A. Margiotta, M. Sioli, and M. Spurio, "A parameterisation of single and multiple muons in the deep water or ice," Astroparticle Physics, vol. 25, no. 1, pp. 1-13, 2006.

[16] D. Heck, "The air shower simulation program CORSIKA and hadronic interaction models," Nachrichten-Karlsruhe, vol. 33, pp. 113-120, 2001.

[17] G. Barr, T. K. Gaisser, and T. Stanev, "Flux of atmospheric neutrinos," Physical Review D, vol. 39, no. 11, pp. 3532-3534, 1989.

[18] V. Agrawal, T. K. Gaisser, P. Lipari, and T. Stanev, "Atmospheric neutrino flux above $1 \mathrm{GeV}$," Physical Review D, vol. 53, no. 3, pp. 1314-1323, 1996.

[19] M. Honda, T. Kajita, K. Kasahara, and S. Midorikawa, "New calculation of the atmospheric neutrino flux in a threedimensional scheme," Physical Review D, vol. 70, no. 4, Article ID 043008, 17 pages, 2004.

[20] J. A. Aguilar, A. Albert, P. Amram et al., "Transmission of light in deep sea water at the site of the ANTARES neutrino telescope," Astroparticle Physics, vol. 23, no. 1, pp. 131-155, 2005.
[21] J. A. Aguilar, I. Al Samarai, A. Albert et al., "A fast algorithm for muon track reconstruction and its application to the ANTARES neutrino telescope," Astroparticle Physics, vol. 34, no. 9, pp. 652$662,2011$.

[22] S. Adrian-Martinez, I. Al Samarai, A. Albert et al., "Measurement of atmospheric neutrino oscillations with the ANTARES neutrino telescope," Physics Letters B, vol. 714, no. 2-5, pp. 224230, 2012.

[23] R. Abbasi, M. Ackermann, J. Adams et al., "The IceCube data acquisition system: signal capture, digitization, and timestamping," Nuclear Instruments and Methods in Physics Research A, vol. 601, no. 3, pp. 294-316, 2009.

[24] R. Abbasi, Y. Abdou, T. Abu-Zayyad et al., "Calibration and characterization of the IceCube photomultiplier tube," Nuclear Instruments and Methods in Physics Research A, vol. 618, no. 1-3, pp. 139-152, 2010.

[25] M. G. Aartsen, R. Abbasi, Y. Abdou et al., "Measurement of atmospheric neutrino oscillations with IceCube," Physical Review Letters, vol. 111, no. 8, Article ID 081801, 6 pages, 2013.

[26] O. Schulz and The IceCube collaboration, in Proceedings of the 31st International Cosmic Ray Conference (ICRC '09), Łódź, Poland, 2009.

[27] J. Ahrens, X. Bai, R. Bay et al., "Muon track reconstruction and data selection techniques in AMANDA," Nuclear Instruments and Methods in Physics Research A, vol. 524, no. 1-3, pp. 169194, 2004.

[28] Ch. Wiebusch and The IceCube collaboration, in Proceedings of the 33rd International Cosmic Ray Conference (ICRC '13), Rio de Janeiro, Brazil, 2013, contribution 0848.

[29] J. P. Yanez and The IceCube collaboration, in Proceedings of the 33rd International Cosmic Ray Conference (ICRC '13), Rio de Janeiro, Brazil, 2013, contribution 0450.

[30] P. Adamson, C. Andreopoulos, R. Armstrong et al., "Measurement of the neutrino mass splitting and flavor mixing by MINOS," Physical Review Letters, vol. 106, no. 18, Article ID 181801, 6 pages, 2011.

[31] K. Abe, Y. Hayato, T. Iida et al., "Search for differences in oscillation parameters for atmospheric neutrinos and antineutrinos at super-kamiokande," Physical Review Letters, vol. 107, no. 24, Article ID 241801, 6 pages, 2011.

[32] G. L. Fogli, E. Lisi, A. Marrone, D. Montanino, A. Palazzo, and A. M. Rotunno, "Global analysis of neutrino masses, mixings, and phases: entering the era of leptonic $C P$ violation searches," Physical Review D, vol. 86, no. 1, Article ID 013012, 10 pages, 2012.

[33] V. D. Barger, S. Geer, R. Raja, and K. Whisnant, "Long-baseline study of the leading neutrino oscillation at a neutrino factory," Physical Review D, vol. 62, no. 1, Article ID 013004, 14 pages, 2000.

[34] P. Huber, M. Lindner, and W. Winter, "Simulation of longbaseline neutrino oscillation experiments with GLoBES: (general long baseline experiment simulator)," Computer Physics Communications, vol. 167, no. 3, pp. 195-202, 2005.

[35] P. Huber, J. Kopp, M. Lindner, M. Rolinec, and W. Winter, "New features in the simulation of neutrino oscillation experiments with GLoBES 3.0: (general long baseline experiment simulator)," Computer Physics Communications, vol. 177, no. 5, pp. 432438, 2007.

[36] A. M. Dziewonski and D. L. Anderson, "Preliminary reference earth model," Physics of the Earth and Planetary Interiors, vol. 25, no. 4, pp. 297-356, 1981. 
[37] C. Andreopoulos, A. Bell, D. Bhattacharya et al., "The GENIE neutrino Monte Carlo generator," Nuclear Instruments and Methods in Physics Research A, vol. 614, no. 1, pp. 87-104, 2010.

[38] E. Kh. Akhmedov, S. Razzaque, and A. Yu. Smirnov, "Mass hierarchy, 2-3 mixing and CP-phase with huge atmospheric neutrino detectors," Journal of High Energy Physics, vol. 2013, article 82, 2013.

[39] KM3NeT, Technical Design Report for a Deep-sea Research Infrastructure in the Mediterranean Sea Incorporating a Very Large Volume Neutrino Telescope, 2010, http://www.km3net.org.

[40] P. Kooijman and The KM3Net Collaboration, "The Neutrino Mass Hierarchy in KM3Net-ORCA, a feasibility study," in Proceedings of 33rd International Cosmic Ray Conference (ICRC '13), Rio de Janeiro, Brazil, 2013, contribution 0164.

[41] A. Gross and The IceCube collaboration, in Proceedings of the 33rd International Cosmic Ray Conference (ICRC '13), Rio de Janeiro, Brazil, 2013, contribution 0555.

[42] W. Winter, "Neutrino mass hierarchy determination with IceCube-PINGU," Physical Review D, vol. 88, no. 1, Article ID 013013, 13 pages, 2013.

[43] M. Ribordy and A. Yu. Smirnov, "Improving the neutrino mass hierarchy identification with inelasticity measurement in PINGU and ORCA," Physical Review D, vol. 87, no. 11, Article ID 113007, 20 pages, 2013.

[44] C. Lujan-Peschard, G. Pagliaroli, and F. Vissani, "Counting muons to probe the neutrino mass spectrum," The European Physical Journal C, vol. 73, article 2439, 2013.

[45] J. Brunner, "Counting electrons to probe the Neutrino Mass Hierarchy," http://arxiv.org/abs/1304.6230.

[46] Google Maps, http://maps.google.com.

[47] V. V. Ammosov, S. V. Belikov, A. P. Bugorsky et al., "A study of $v_{e}-v_{\mu}$ universality and search for neutrino oscillations," Zeitschrift für Physik C, vol. 40, no. 4, pp. 487-491, 1988.

[48] R. B. Patterson, "The NOvA experiment: status and outlook," Nuclear Physics B, vol. 235-236, pp. 151-157, 2013.

[49] Y. S. Jeong and M. H. Reno, "Tau neutrino and antineutrino cross sections," Physical Review D, vol. 82, Article ID 033010, 6 pages, 2010 .

[50] R. Nahnhauer, Habilitation, Humboldt-University, Berlin, Germany, (unpublished, private communication). 

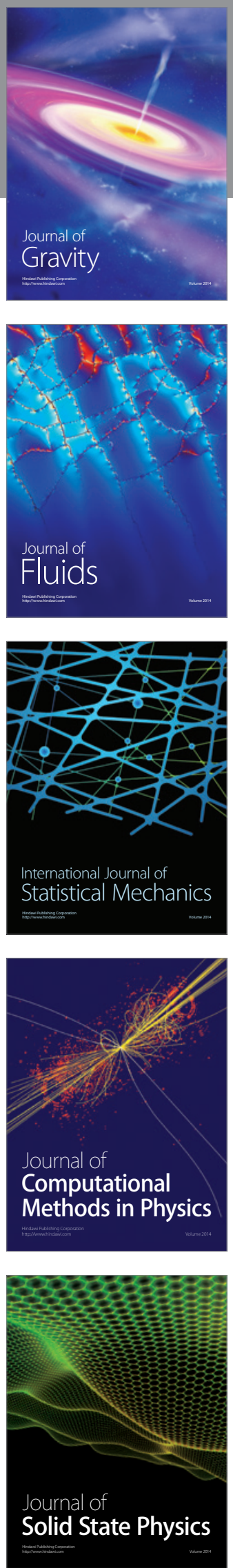

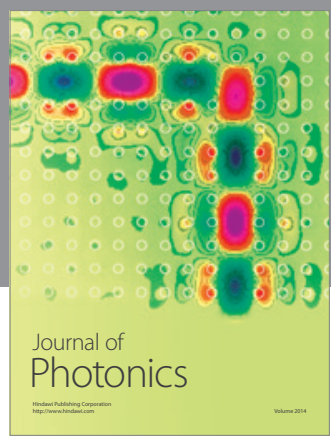

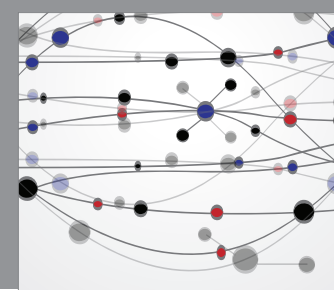

The Scientific World Journal

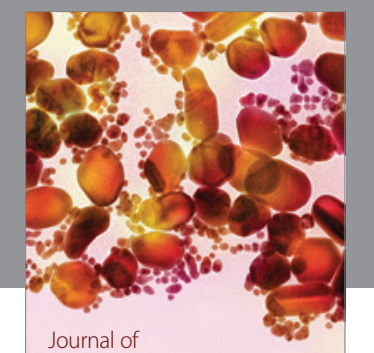

Soft Matter
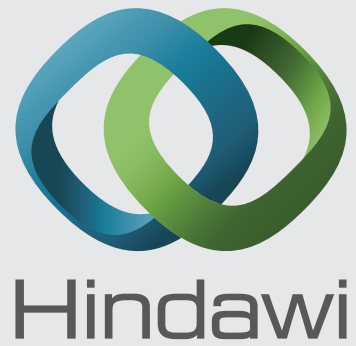

Submit your manuscripts at

http://www.hindawi.com
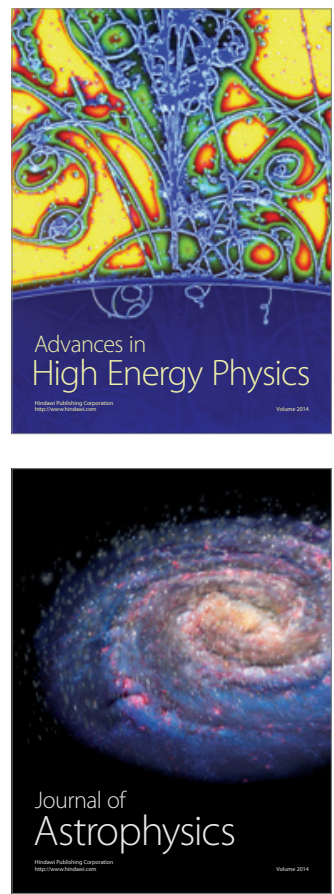
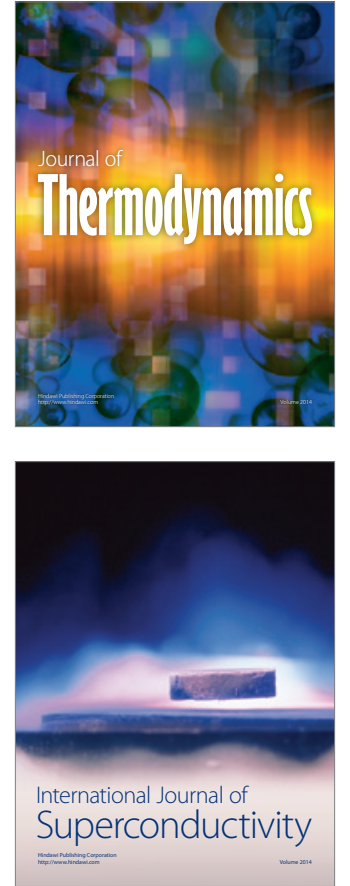
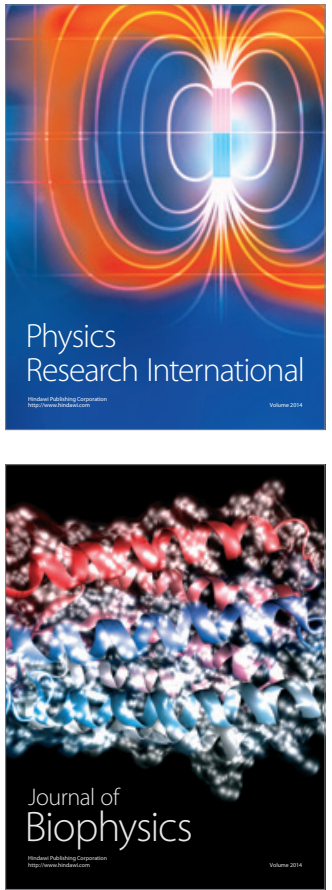
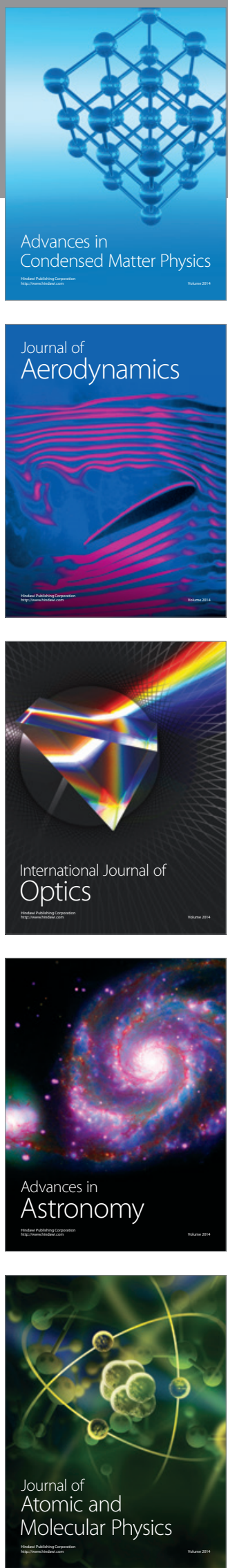